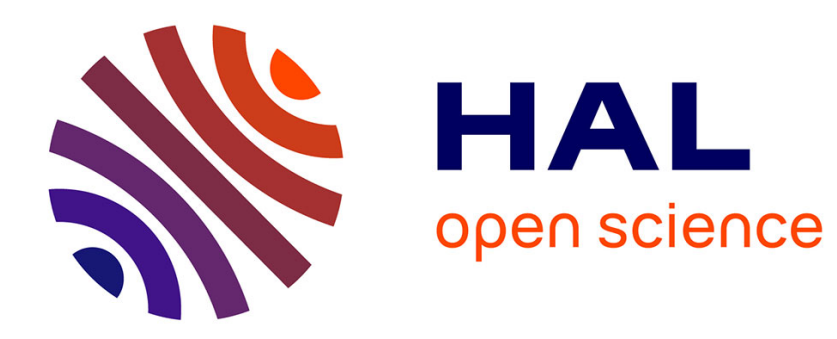

\title{
Dirichlet eigenvalues of asymptotically flat triangles
}

Thomas Ourmières-Bonafos

\section{- To cite this version:}

Thomas Ourmières-Bonafos. Dirichlet eigenvalues of asymptotically flat triangles. Asymptotic Analysis, 2015, 92 (3-4), pp.279-312. 10.3233/ASY-1412792015 . hal-00958748v2

\section{HAL Id: hal-00958748 \\ https://hal.science/hal-00958748v2}

Submitted on 17 Nov 2014

HAL is a multi-disciplinary open access archive for the deposit and dissemination of scientific research documents, whether they are published or not. The documents may come from teaching and research institutions in France or abroad, or from public or private research centers.
L'archive ouverte pluridisciplinaire HAL, est destinée au dépôt et à la diffusion de documents scientifiques de niveau recherche, publiés ou non, émanant des établissements d'enseignement et de recherche français ou étrangers, des laboratoires publics ou privés. 


\title{
DIRICHLET EIGENVALUES OF AS YMPTOTICALLY FLAT TRIANGLES
}

\author{
THOMAS OURMIÈRES-BONAFOS*
}

\begin{abstract}
This paper is devoted to the study of the eigenpairs of the Dirichlet Laplacian on a family of triangles where two vertices are fixed and the altitude associated with the third vertex goes to zero. We investigate the dependence of the eigenvalues on this altitude. For the first eigenvalues and eigenfunctions, we obtain an asymptotic expansion at any order at the scale cube root of this altitude due to the influence of the Airy operator. Asymptotic expansions of the eigenpairs are provided, exhibiting two distinct scales when the altitude tends to zero. In addition, we generalize our analysis to the case of a shrinking polygon.
\end{abstract}

\section{Introduction}

\subsection{Motivations and related questions}

There are few planar domains for which we can have an explicit expression of the eigenpairs of the Dirichlet Laplacian. Nevertheless there has been a recent interest about it on thin domains in $\mathbb{R}^{2}$. In this limit, asymptotic expansions of the eigenvalues and eigenfunctions can be provided, which informs about the spectrum of the Dirichlet Laplacian.

In this spirit, Borisov and Freitas give in [4] a construction of quasimodes of the Dirichlet Laplacian expanded at any order in the height paramater on thin smooth planar domains. In [11], Friedlander and Solomyak overcome the smooth domain hypothesis: they provide a two-term asymptotics using the convergence of resolvents.

The result of Friedlander and Solomyak applies to triangles but, before investigating triangles in the thin limit, one can cite the work of McCartin [21] who gives an explicit expression of the first eigenvalue of the Dirichlet Laplacian, say $\mu_{1}$, on an equilateral triangle of altitude $H: \mu_{1}(H)=4 \pi^{2} H^{-2}$. Hillairet and Judge prove in [14] the simplicity of the eigenvalues for almost every Euclidean triangle of $\mathbb{R}^{2}$. In [19], Lu and Rowlett are interested in the fundamental gap of "short" triangles.

The question of an asymptotic expansion for the Dirichlet Laplacian on thin triangles has already been studied by Freitas in [10]. In this paper a finite asymptotic expansion of the first two eigenvalues is provided for a family of near isosceles triangles. We also refer to the work of Dauge and Raymond [7] in which an asymptotics at any order is given for the first eigenvalues of the Dirichlet Laplacian on a right-angled thin triangle.

* IRMAR, Univ. Rennes 1, CNRS, Campus de Beaulieu, F-35042 Rennes cedex, France thomas.ourmieres@univ-rennes.1.fr 
The question of finding an asymptotic expansion at any order for the Dirichlet Laplacian eigenmodes on a non-smooth thin domain is still open. We tackle the question in this paper for triangles of altitude $h$ in the regime $h \rightarrow 0$. In a first step we construct quasimodes involving simultaneously two scales: $h^{2 / 3}$ and $h$. The scale $h^{2 / 3}$ is due to the singularity of type $(x \mapsto|x|)$. For smooth domains, in the case of a non-degenerate maximum, the scale which plays the same role is $h$ (see Theorem 1 in [4]). In fact, in our case, there is also a boundary layer of scale $h$ but this scale is not visible at first orders in the eigenpair expansions. That is why in the eigenvalue expansions of [10] and [11] this scale does not appear. Nevertheless it is present in the right-angled case exposed in [7] or in the case of a small apertured cones (see [22]). One of the motivations for this paper is to understand this boundary layer. Is it, for right-angled triangles or cones, induced by the Dirichlet boundary conditions?

In a second step, we get the separation of eigenvalues thanks to the Feshbach method, the associated eigenfunctions being localized near the altitude providing the most space. Unlike the resolvent convergence method exposed in [11] we use Agmon localization estimates which allows to consider cases with multiple peaks.

\subsection{The Dirichlet Laplacian}

Let us denote by $\left(x_{1}, x_{2}\right)$ the Cartesian coordinates of the space $\mathbb{R}^{2}$ and by $\mathbf{0}=(0,0)$ the origin. The positive Laplace operator is given by $-\partial_{1}^{2}-\partial_{2}^{2}$. Let $s \in \mathbb{R}$ and $h>0$ we define $\widehat{\operatorname{Tr}}(s, h)$, the convex hull of the points of coordinates $A=(-1,0), B=(1,0)$ and $C=(s, h)$. We are interested in the eigenvalues of the Dirichlet Laplacian $-\Delta_{\widehat{T r i}(s, h)}^{\operatorname{Dir}}:=-\partial_{1}^{2}-\partial_{2}^{2}$ on the triangle $\widehat{\operatorname{Tr}}(s, h)$ in the regime $h \rightarrow 0$.
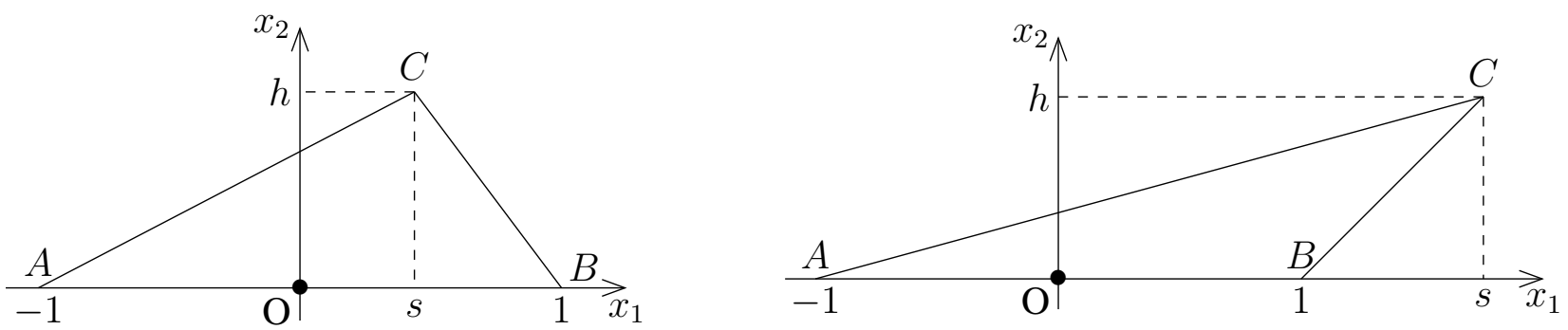

Figure 1: The triangle $\widehat{\operatorname{Tri}}(s, h)$ in the acute and the obtuse configuration

The values of $s$ define different configurations for the geometry of the triangle $\widehat{\operatorname{Tri}}(s, h)$ :

$s=0$ corresponds to isoscele triangles,

$|s|<1$ corresponds to acute triangles (see Figure 1)

$|s|=1$ corresponds to right-angled triangles,

$|s|>1$ corresponds to obtuse triangles (see Figure 1).

Since $\widehat{\operatorname{Tr}}(s, h)$ is convex, the domain of the operator $-\Delta \widehat{\operatorname{Tri}(s, h)} \underset{\mathrm{Dir}}{\text { is }}$ the space of functions in $H^{2}(\widehat{\operatorname{Tri}}(s, h)) \cap H_{0}^{1}(\widehat{\operatorname{Tri}}(s, h))$ (see [17]). Since $\widehat{\operatorname{Tri}}(s, h)$ is a bounded domain $-\Delta_{\widehat{\operatorname{Tri}}(s, h)}^{\mathrm{Dir}}$ has compact resolvent and its spectrum is a non-decreasing sequence of eigenvalues denoted $\left(\mu_{n}(s, h)\right)_{n \geq 1}$. 


\subsection{First properties of the eigenvalues}

Before stating the main results of this paper, one can notice that in the obtuse configuration of Figure 1 the regime $h \rightarrow 0$ is equivalent to the one where the altitude from $B$ goes to zero. In fact, for $s>1$, the length of the line segment $[A C]$ is $\left((1+s)^{2}+h^{2}\right)^{1 / 2}$. Let us perform the dilatation

$$
\tilde{x}_{1}=\frac{2}{\sqrt{(1+s)^{2}+h^{2}}} x_{1} ; \quad \tilde{x}_{2}=\frac{2}{\sqrt{(1+s)^{2}+h^{2}}} x_{2} .
$$

If we denote by $\tilde{A}, \tilde{B}$ and $\tilde{C}$ the image of $A, B$ and $C$ through the dilatation, the line segment $[A C]$ is transformed into the line segment $[\tilde{A} \tilde{C}]$ of length 2 and $-\Delta \underset{\text { Tri }(s, h)}{\mathrm{Dir}}$ is unitarily equivalent to

$$
\frac{(s+1)^{2}+h^{2}}{4}\left(-\partial_{\tilde{x}_{1}}^{2}-\partial_{\tilde{x}_{2}}^{2}\right)
$$

After a rotation and a translation, the triangle $\tilde{A} \tilde{B} \tilde{C}$ can be seen as $\widehat{\operatorname{Tri}}(\tilde{s}, \tilde{h})$, where we have

$$
\tilde{s}=\frac{s}{\sqrt{(1+s)^{2}+h^{2}}} ; \quad \tilde{h}=\frac{2 h}{\sqrt{(1+s)^{2}+h^{2}}},
$$

$\tilde{h}$ being the altitude from $\tilde{B}$. We have

$$
0<\tilde{s}<1, \quad \tilde{h} \underset{h \rightarrow 0}{\longrightarrow} 0
$$

and we get

$$
\mu_{n}(s, h)=\frac{(s+1)^{2}+h^{2}}{4} \mu_{n}(\tilde{s}, \tilde{h}) .
$$

The same kind of computations can be done for $s<-1$, so that, we can take $s \in(-1,1)$.

At fixed $h$, the question of the regularity of $\mu_{n}(\cdot, h)$ in $|s|=1$ is considered in Section 2 but one can see that thanks to a Dirichlet bracketing (see [23, Chap. XIII]) we have, for $s$ in a left neighborhood of 1 :

$$
\mu_{n}\left(1, \frac{2 h}{1+s}\right) \leq \mu_{n}(s, h) \leq \frac{4}{(1+s)^{2}} \mu_{n}\left(1, \frac{2 h}{1+s}\right)
$$

Since $\mu_{n}(1, \cdot)$ is continuous for all $h>0$ we obtain the left continuity of $\mu_{n}(\cdot, h)$ in $s=1$. We can apply the same reasoning for the right continuity and we obtain the continuity of $\mu_{n}(\cdot, h)$ in $s=1$.

We have the following lower bound for $\mu_{n}(s, h)$ :

Proposition 1.1 For all $s \in(-1,1)$ and $h>0$, we have:

$$
\frac{\pi^{2}}{h^{2}}+\frac{\pi^{2}}{4} \leq \mu_{n}(s, h)
$$

Proof: The triangle $\widehat{\operatorname{Tr}}(s, h)$ is included in the rectangle $(-1,1) \times(0, h)$ and the conclusion follows by Dirichlet bracketing. 


\subsection{Schrödinger operators in one dimension}

In the analysis of $-\Delta \underset{\operatorname{Tri}(s, h)}{\mathrm{Dir}}$ a one dimensional operator, constructed in the spirit of the Born-Oppenheimer approximation (see [5, 16, 20]), plays an important role: By replacing $-\partial_{x_{2}}^{2}$ in the expression of $-\Delta_{\widehat{T r i}(s, h)}^{\mathrm{Dir}}$ by its lowest eigenvalue on each slice of $\widehat{\operatorname{Tr}}(s, h)$ at fixed $x_{1}$ we obtain an effective potential $v_{s}$ and, on $L^{2}(-1,1)$, we arrive to the operator :

$$
-\partial_{x_{1}}^{2}+h^{-2} v_{s}\left(x_{1}\right), \quad \text { with } v_{s}\left(x_{1}\right)= \begin{cases}\frac{(1+s)^{2}}{\left(1+x_{1}\right)^{2}} \pi^{2}, & \text { for }-1<x_{1}<s, \\ \frac{(1-s)^{2}}{\left(1-x_{1}\right)^{2}} \pi^{2}, & \text { for } s<x_{1}<1 .\end{cases}
$$

When $h \rightarrow 0$, we know that the minimum of the effective potential $v_{s}$ guides the behavior of the ground eigenpairs of $-\Delta_{\widehat{T r i}(s, h)}^{\mathrm{Dir}}\left(\right.$ see [6, Chap. 11], [8] and [24]). This minimum is attained at $x_{1}=s$. In a neighborhood of $x_{1}=s, v_{s}$ can be approximated by its left and right tangents, it yields the operator $l_{s}^{\tan }(h):=-\partial_{x_{1}}^{2}+h^{-2} v_{s}^{\tan }\left(x_{1}\right), \quad$ with $v_{s}^{\tan }\left(x_{1}\right)=\pi^{2}+2 \pi^{2}\left(\frac{1}{1+s} \mathbb{1}_{x_{1}<s}\left(x_{1}\right)+\frac{1}{1-s} \mathbb{1}_{x_{1}>s}\left(x_{1}\right)\right)\left|x_{1}-s\right|$

To arrive to a canonical form, we perform the scaling $x_{1}=\frac{h^{2 / 3}}{\sqrt[3]{2 \pi^{2}}} u+s$ and we have:

$$
l_{s}^{\tan }(h) \sim h^{-2} \pi^{2}+\left(2 \pi^{2}\right)^{2 / 3} h^{-4 / 3} l_{s}^{\bmod }\left(u ; \partial_{u}\right),
$$

where the model operator $l_{s}^{\text {mod }}$ is defined, on $L^{2}(\mathbb{R})$, as:

$$
l_{s}^{\bmod }\left(u ; \partial_{u}\right):=-\partial_{u}^{2}+v_{s}^{\bmod }(u), \quad \text { with } v_{s}^{\bmod }(u):=\left(\frac{1}{1+s} \mathbb{1}_{\mathbb{R}_{-}}(u)+\frac{1}{1-s} \mathbb{1}_{\mathbb{R}_{+}}(u)\right)|u|,
$$

where $\operatorname{Dom}\left(l_{s}^{\text {mod }}\right)=\left\{\Psi \in H^{2}(\mathbb{R}): u \Psi \in L^{2}(\mathbb{R})\right\}$. The parameter $s$ introduces a skewness in the effective potential $v_{s}^{\text {mod }}$. We will see in Section 2 that this model operator is related to the Airy functions. We remark that for $s>1$ the roles of $s$ and 1 are interverted. In fact, the Born-Oppenheimer strategy yields, on $L^{2}(-1, s)$, the operator:

$$
-\partial_{x_{1}}^{2}+h^{-2} \hat{v}_{s}\left(x_{1}\right), \quad \text { with } \hat{v}_{s}\left(x_{1}\right)= \begin{cases}\frac{(1+s)^{2}}{\left(1+x_{1}\right)^{2}} \pi^{2}, & \text { for }-1<x_{1}<1, \\ \frac{\left(s^{2}-1\right)^{2}}{4\left(s-x_{1}\right)^{2}} \pi^{2}, & \text { for } 1<x_{1}<s .\end{cases}
$$

The tangent operator writes as

$\hat{l}_{s}^{\tan }:=-\partial_{x_{1}}^{2}+h^{-2} \hat{v}_{s}^{\tan }\left(x_{1}\right), \quad$ with $\hat{v}_{s}^{\tan }\left(x_{1}\right)=\frac{(1+s)^{2}}{4}\left(\pi^{2}+2 \pi^{2}\left(\frac{1}{2} \mathbb{1}_{x_{1}<1}+\frac{1}{s-1} \mathbb{1}_{x_{1}>1}\right)|u-1|\right)$.

To arrive to a canonical form, we perform the scaling $x_{1}=\left(\frac{(1+s)^{2}}{2} \pi^{2}\right)^{-1 / 3} h^{2 / 3} \hat{u}+1$ and we have:

$$
\hat{l}_{s}^{\tan } \sim h^{-2} \frac{(1+s)^{2}}{4} \pi^{2}+\left(\frac{(1+s)^{2}}{2} \pi^{2}\right)^{2 / 3} h^{-4 / 3} \hat{l}_{s}^{\bmod }\left(\hat{u}, \partial_{\hat{u}}\right)
$$

where the model operator $\hat{l}_{s}^{\text {mod }}$, defined on $L^{2}(\mathbb{R})$, as:

$$
\hat{l}_{s}^{\text {mod }}:=-\partial_{\hat{u}}^{2}+\hat{v}_{s}^{\text {mod }}(\hat{u}), \quad \text { with } \hat{v}_{s}^{\text {mod }}(\hat{u}):=\left(\frac{1}{2} \mathbb{1}_{\mathbb{R}_{-}}(\hat{u})+\frac{1}{s-1} \mathbb{1}_{\mathbb{R}_{+}}(\hat{u})\right)|\hat{u}| .
$$




\subsection{Asymptotic expansion of eigenvalues}

We recall that $\mu_{n}(s, h)$ is the $n^{- \text {th }}$ eigenvalue of the Dirichlet Laplacian $-\Delta_{\widehat{T r i}(s, h)}^{\mathrm{Dir}}$ on the geometrical domain $\widehat{\operatorname{Tr}}(s, h)$. The main result of this paper is an asymptotic expansion of the eigenvalues $\mu_{n}(s, h)$ as $h \rightarrow 0$. Indeed, the lowest eigenvalues of $-\Delta_{\widehat{T r i}(s, h)}^{\operatorname{Dir}}$ admit expansions at any order in power of $h^{1 / 3}$. In the proof we also provide the structure of the eigenfunctions associated with these eigenvalues: at first order they are, up to some constants, almost a tensor product between the first eigenfunction of the Dirichlet Laplacian in the transverse variable $x_{2}$ and, up to normalization constants, the eigenfunctions of the model operator $l_{s}^{\text {mod }}$ in the $x_{1}$ variable. Moreover they are localized near the altitude from $C$.

Theorem 1.2 Let $0<s_{0}<1$. For all $s \in\left[-s_{0}, s_{0}\right]$, the eigenvalues of $-\Delta \underset{T \mathrm{Tr}(s, h)}{\mathrm{Dir}}$, denoted by $\mu_{n}(s, h)$, admit the expansions:

$$
\mu_{n}(s, h) \underset{h \rightarrow 0}{\sim} h^{-2} \sum_{j \geq 0} \beta_{j, n}(s) h^{j / 3},
$$

uniformly in $s$ (see Notation 1.3). The functions $\left(s \mapsto \beta_{j, n}(s)\right)$ are analytic on $(-1,1)$ and we have: $\beta_{0, n}=\pi^{2}, \beta_{1, n}=0$ and $\beta_{2, n}(s)=\left(2 \pi^{2}\right)^{2 / 3} \kappa_{n}(s)$, where the $\kappa_{n}(s)$ are the eigenvalues of the model operator defined in (1.3). Moreover the eigenfunctions contains simultaneously the two scales $h^{2 / 3}$ and $h$ as it can be seen in equation (3.10).

Notation 1.3 Let $\Lambda(s, h)$ be a function of $s$ and $h$ and let $\theta>0$. We say that $\Lambda(s, h) \underset{h \rightarrow 0}{\sim} \sum_{j \geq 0} \Gamma_{j}(s) h^{j \theta}$ if, for all $J \in \mathbb{N}$, there exists $C_{J}(s)>0$ and $h_{0}(s)>0$, such that for all $h \in\left(0, h_{0}(s)\right)$

$$
\left|\Lambda(s, h)-\sum_{j=0}^{J} \Gamma_{j}(s) h^{\theta j}\right| \leq C_{J}(s) h^{\theta(J+1)} .
$$

We say that the asymptotic expansion is uniform in $s$ if $C_{J}(s)$ and $h_{0}(s)$ do not depend on $s$.

\subsection{The mountain with two peaks}

Thanks to the structure of the proof of Theorem 1.2, we can understand the spectrum of the Dirichlet Laplacian on a thin shrinking polygon. Let us choose $s^{\text {lef }}, s^{\text {rig }} \in(0,1), \tau \in(0,1], \varsigma \in(0, \tau)$ and let $h>0$, we consider in $\mathbb{R}^{2}$ the points $A=(-1,0), B=(1,0), C_{1}=\left(s^{\text {rig }}, h\right), C_{2}=\left(-s^{\text {lef }}, \tau h\right)$ and $D=(0, \varsigma)$. Let $\Omega^{\text {rig }}(h)$ be the open convex hull of $\mathbf{0}, B, C_{1}$ and $D$ and $\Omega^{\text {lef }}(h)$ the open convex hull of $\mathbf{0}, A, C_{2}$ and $D$. Then, we define $\Omega(h)$ (see Figure 2) by:

$$
\Omega(h)=\Omega^{\text {rig }}(h) \cup \Omega^{\text {lef }}(h) \cup[\mathbf{0}, D] .
$$

Let $\left(\mu_{n}(h)\right)_{n \geq 1}$ be the non-decreasing sequence of eigenvalues of $-\Delta_{\Omega(h)}^{\mathrm{Dir}}$, the Dirichlet realization of the Laplacian on $\Omega(h)$.

We consider the Dirichlet realizations of the Laplacian on $\Omega^{\text {lef }}(h)$ and $\Omega^{\text {rig }}(h)$, respectively denoted by $-\Delta_{\Omega^{\operatorname{lef}}(h)}^{\mathrm{Dir}}$ and $-\Delta_{\Omega^{\mathrm{rig}}(h)}^{\mathrm{Dir}}$. We respectively denote by $\left(\mu_{n}^{\text {lef }}(h)\right)_{n \geq 1}$ and $\left(\mu_{n}^{\text {rig }}(h)\right)_{n \geq 1}$ their eigenvalues.

Let us define the operator $\mathfrak{D}(h):=-\Delta_{\Omega^{\text {lef }}(h)}^{\mathrm{Dir}} \oplus-\Delta_{\Omega^{\text {rig }}(h)}^{\mathrm{Dir}}$. We denote by $\left(\nu_{n}(h)\right)_{n \geq 1}$ its eigenvalues counted with multiplicity. In fact, each eigenpair of $\mathfrak{D}(h)$ is associated with either an eigenpair of $-\Delta_{\Omega^{\text {lef }}(h)}^{\mathrm{Dir}}$ or $-\Delta_{\Omega^{\text {rig }(h)}}^{\mathrm{Dir}}$ and reciprocally. More precisely, for $k \in \mathbb{N}^{*}$ we consider the eigenpairs 


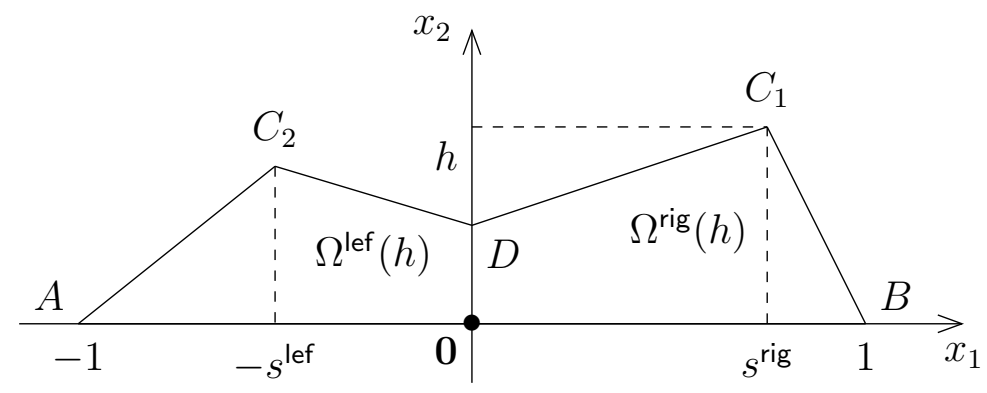

Figure 2: The domain $\Omega(h)$

$\left(\mu_{k}^{\text {lef }}(h), \psi_{k, h}^{\text {lef }}\right)$ and $\left(\mu_{k}^{\text {rig }}(h), \psi_{k, h}^{\text {rig }}\right)$ respectively of $-\Delta_{\Omega^{\text {lef }}(h)}^{\text {Dir }}$ and $-\Delta_{\Omega^{\text {rig }}(h)}^{\text {Dir }}$. By definition of $\mathfrak{D}(h)$, there exists $n_{k}^{\text {lef }}(h), n_{k}^{\text {rig }}(h) \in \mathbb{N}^{*}$ such that:

$$
\nu_{n_{k}^{\text {lef }}(h)}(h)=\mu_{k}^{\text {lef }}(h) ; \quad \nu_{n_{k}^{\text {rig }}(h)}(h)=\mu_{k}^{\text {rig }}(h) .
$$

Moreover if $\psi_{k, h}^{\text {lef }}$ (respectively $\psi_{k, h}^{\text {rig }}$ ) is the eigenfunction associated with $\mu_{k}^{\text {lef }}(h)\left(\right.$ respectively $\mu_{k}^{\text {rig }}(h)$ ) then, $\left(\psi_{k, h}^{\text {lef }}, 0\right)$ (respectively $\left.\left(0, \psi_{k, h}^{\text {rig }}\right)\right)$ is the eigenfunction of $\mathfrak{D}(h)$ associated with $\nu_{n_{k}^{\text {lef }}(h)}(h)$ (respectively $\nu_{n_{k}^{\text {rig }}(h)}(h)$ ). Furthermore the sequences $\left(n_{k}^{\text {lef }}(h)\right)_{k \in \mathbb{N}^{*}}$ and $\left(n_{k}^{\text {rig }}(h)\right)_{k \in \mathbb{N}^{*}}$ are increasing, have disjoint images and verify $\mathbb{N}^{*}=\left\{n_{k}^{\text {lef }}(h)\right\}_{k \in \mathbb{N}^{*}} \cup\left\{n_{k}^{\text {rig }}(h)\right\}_{k \in \mathbb{N}^{*}}$.

We will prove

Theorem 1.4 For all $N \in \mathbb{N}^{*}$ there exist $h_{0}>0, C_{0}>0$ and $C>0$ such that for all $h \in\left(0, h_{0}\right)$ and all $j \in\{1, \ldots, N\}$ :

$$
\left|\mu_{j}(h)-\nu_{j}(h)\right| \leq C_{0} e^{-C / h} .
$$

As explained for the triangle, in the regime $h \rightarrow 0$ the eigenfunctions are localized near the altitude providing the most space. Here they are localized near the altitude from $C_{1}, C_{2}$ or both. In this last case, they interact at an exponentially small scale.

\subsection{Structure of the paper}

In Section 2 we study the model operator defined in (1.3). We describe its eigenvalues and their dependence on the parameter $s$. In Section 3, we perform a change of variables that transforms the triangle into a rectangle. The operator is more complicated but we deal with a simpler geometrical domain. Thanks to this change of variables and some lemmas derived from the Fredholm alternative we can construct quasimodes. We finish the proof of Theorem 1.2 using a Feshbach-Grushin projection which justifies that the model operator is an actual approximation of our problem. Then we get the separation of the eigenvalues. In Section 4 we study the mountain $\Omega(h)$. We use properties derived from Theorem 1.2 to prove Theorem 1.4.

We conclude by Appendix A by providing numerical experiments which agree with the theoretical shape of the eigenfunctions.

\section{Model operator}

To deal with $-\Delta_{T \text { Tri }(s, h)}^{\text {iir }}$ we first need to study some basic properties of the model operator $l_{s}^{\text {mod }}$. The difference with the operator studied by Dauge and Raymond in [7, Sec. 3] is that the model operator 
is not, up to a constant, the Airy reversed operator with Dirichlet boundary condition at $u=0$. The effective potential of $l_{s}^{\text {mod }}$ is a combination on $\mathbb{R}_{-}$of an Airy reversed operator and on $\mathbb{R}_{+}$of an Airy operator. The non-symmetry of this potential and the transmission conditions at $u=0$ complicate the study: we cannot have an explicit expression of the eigenvalues of $l_{s}^{\mathrm{mod}}$ as zeros of an Airy function. The basic definitions and properties of the Airy functions, that we use in this paper, can be found in [1].

The behavior of the effective potential $v_{s}^{\text {mod }}$ when $|u| \rightarrow+\infty$ yields the

Proposition 2.1 For all $s \in(-1,1)$, the model operator $l_{s}^{\text {mod }}$ has compact resolvent.

Thus, the spectrum of the model operator $l_{s}^{\text {mod }}$ consists in a non-decreasing sequence of eigenvalues denoted $\left(\kappa_{n}(s)\right)_{n \geq 1}$.

Remark 2.2 When $s=0$, the model operator is $l_{0}^{\bmod }\left(u ; \partial_{u}\right)=-\partial_{u}^{2}+|u|$ and its eigenvalues are, for all $n \geq 0$ :

$$
\left\{\begin{array}{l}
\kappa_{2 n+1}(0)=z_{\mathrm{A}}^{\prime}(n+1), \\
\kappa_{2 n+2}(0)=z_{\mathrm{A}}(n+1),
\end{array}\right.
$$

where, for all $k \in \mathbb{N}^{*}, z_{\mathrm{A}}(k)$ and $z_{\mathrm{A}}^{\prime}(k)$ are respectively the zeros of the Airy reversed function and the zeros of the first derivative of the Airy reversed function.

Thanks to the theory about Sturm-Liouville operators, we get

Proposition 2.3 For all $s \in(-1,1)$, the eigenvalues of the model operator $l_{s}^{\text {mod }}$ are simple.

Now, we are interested in the regularity of these eigenvalues. The family $\left(l_{s}^{\text {mod }}\right)_{s \in(-1,1)}$ is an analytic family of type (A) (see [15]). Jointly with Proposition 2.3, we have the

Proposition 2.4 For all $n \geq 1$, the functions $\left(s \rightarrow \kappa_{n}(s)\right)$ are analytic on $(-1,1)$. Moreover for all $n \in \mathbb{N}^{*}$, there exists an eigenfunction $\mathrm{T}_{s}^{n}$ associated with $\kappa_{n}(s)$, such that the functions $\left(s \rightarrow \mathrm{T}_{s}^{n} \in \operatorname{Dom}\left(l_{s}^{\mathrm{mod}}\right)\right)$ are also analytic on $(-1,1)$.

Characterization of the eigenvalues $\left(\kappa_{n}(s)\right)_{n \geq 1} \quad$ We have the

Proposition 2.5 The eigenvalues $\left(\kappa_{n}(s)\right)_{n \geq 1}$ of $l_{s}^{\mathrm{mod}}$ satisfy the following implicit equation in $(s, \kappa)$ :

$$
\sqrt[3]{1+s} \mathrm{~A}\left((1+s)^{2 / 3} \kappa\right) \mathrm{A}^{\prime}\left((1-s)^{2 / 3} \kappa\right)+\sqrt[3]{1-s} \mathrm{~A}\left((1-s)^{2 / 3} \kappa\right) \mathrm{A}^{\prime}\left((1+s)^{2 / 3} \kappa\right)=0,
$$

where $\mathrm{A}$ denotes the Airy reversed function defined by $\mathrm{A}(u)=\mathrm{Ai}(-u)$.

Proof: Let $(\kappa, \Psi)$ be an eigenpair of $l_{s}^{\text {mod }}$. We define:

$$
\Psi^{ \pm}:=\Psi \mathbb{1}_{\mathbb{R}_{ \pm}}
$$

In order to solve

$$
l_{s}^{\bmod } \Psi=\kappa \Psi,
$$

we consider this equation for both $u<0$ and $u>0$. For $u<0$ equation (2.2) writes

$$
\left(-\partial_{u}^{2}-\frac{1}{1+s} u-\kappa\right) \Psi^{-}=0 .
$$


It is an Airy reversed equation. For integrability reasons the Airy function of second kind does not appear in the expression of $\Psi^{-}$and we have:

$$
\Psi^{-}(u)=\alpha^{-} \mathrm{A}\left((1+s)^{-1 / 3}(u+\kappa(1+s))\right),
$$

where $\alpha^{-} \in \mathbb{R}$.

For $u>0$, the same reasoning yields:

$$
\Psi^{+}(u)=\alpha^{+} \operatorname{Ai}\left((1-s)^{-1 / 3}(u-\kappa(1-s))\right),
$$

where $\alpha^{+} \in \mathbb{R}$.

If we denote by $\operatorname{Dom}\left(l_{s}^{\text {mod }}\right)$ the domain of the model operator $l_{s}^{\text {mod }}$, the eigenfunction $\Psi$ belongs to $\operatorname{Dom}\left(l_{s}^{\text {mod }}\right)$. In particular, $\Psi \in H^{2}(\mathbb{R})$ and we have the transmission conditions

$$
\left\{\begin{array}{cc}
\Psi^{-}(0) & =\Psi^{+}(0) \\
\partial_{u} \Psi^{-}(0) & =\partial_{u} \Psi^{+}(0)
\end{array}\right.
$$

which becomes

$$
\left\{\begin{array}{cc}
\alpha^{-} \mathrm{A}\left((1+s)^{2 / 3} \kappa\right)-\alpha^{+} \mathrm{A}\left((1-s)^{2 / 3} \kappa\right) & =0 \\
\alpha^{-}(1-s)^{1 / 3} \mathrm{~A}^{\prime}\left((1+s)^{2 / 3} \kappa\right)+\alpha^{+}(1+s)^{1 / 3} \mathrm{~A}^{\prime}\left((1-s)^{2 / 3} \kappa\right) & =0
\end{array}\right.
$$

The $\kappa_{n}(s)$ are the values for which this system is linked and we get the implicit equation (2.1).

Thanks to the explicit equations (2.3) and (2.4) and the properties of the Airy function of first kind we have the

Proposition 2.6 For all $n \in \mathbb{N}^{*}$ the eigenfunction $\mathrm{T}_{s}^{n}$ belongs to $H_{\mathrm{exp}}^{2}(\mathbb{R})$ (see Notation 2.7 below).

Notation 2.7 For $k \in \mathbb{N}$ and an unbounded domain $\mathcal{D} \subset \mathbb{R}$, we define the spaces

$$
H_{\text {exp }}^{k}(\mathcal{D})=\left\{f \in L^{2}(\mathcal{D}): \exists r>0, \exists \theta>0, \forall p \in\{0, \ldots, k\}, e^{\theta|u|^{r}} f^{(p)} \in L^{2}(\mathcal{D})\right\},
$$

where $f^{(p)}$ is the $p$-th derivative of $f$. For $\mathcal{D} \subset \mathbb{R}^{2}$, unbounded in the $u$ direction, we define the spaces $H_{\text {exp }}^{k}(\mathcal{D})=\left\{f \in L^{2}(\mathcal{D}): \exists r>0 \exists \theta>0, \forall p_{1}, p_{2} \in \mathbb{N}\right.$ such that $\left.p_{1}+p_{2} \leq k, e^{\theta|u|^{r}} \partial_{u}^{p_{1}} \partial_{t}^{p_{2}} f \in L^{2}(\mathcal{D})\right\}$, where we denoted by $t$ the second variable.

To understand the regularity of $\left(s \mapsto \kappa_{n}(s)\right)$ near $s=1$, we perform the change of variables $\sigma=(1-s)^{1 / 3}$ in equation (2.1). It becomes:

$$
\left(2-\sigma^{3}\right)^{1 / 3} \mathrm{~A}\left(\left(2-\sigma^{3}\right)^{2 / 3} \kappa\right) \mathrm{A}^{\prime}\left(\sigma^{2} \kappa\right)+\sigma \mathrm{A}\left(\sigma^{2} \kappa\right) \mathrm{A}^{\prime}\left(\left(2-\sigma^{3}\right)^{2 / 3} \kappa\right)=0,
$$

which is smooth near $\sigma=0$. For $s>1$, the same study with the operator (1.4) gives the implicit equation

$4^{1 / 6} \mathrm{~A}\left(\frac{4}{(s+1)^{4 / 3}} \kappa\right) \mathrm{A}^{\prime}\left(\left(\frac{4(s-1)}{(s+1)^{2}}\right)^{2 / 3} \kappa\right)+(1+s)^{1 / 3}(s-1)^{1 / 3} \mathrm{~A}\left(\left(\frac{4(s-1)}{(s+1)^{2}}\right)^{2 / 3} \kappa\right) \mathrm{A}^{\prime}\left(\frac{4}{(s+1)^{4 / 3}} \kappa\right)=0$

which shows the same behavior of $\kappa_{n}(s)$ for $s>1$. We see that $\kappa_{n}$ is continuous at $s=1$ with $\kappa_{n}(1)=2^{-2 / 3} z_{\mathrm{A}}(n)$. Nevertheless $\kappa_{n}$ has a cubic singularity on the left and on the right of $s=1$. Thanks to the implicit expressions (both for $s<1$ and $s>1$ ) we illustrated on Figure 2 the dependence on $s$ of $\kappa_{n}(s)$ for $n=1,2,3$. It shows the cubic singularity at $s=1$. 


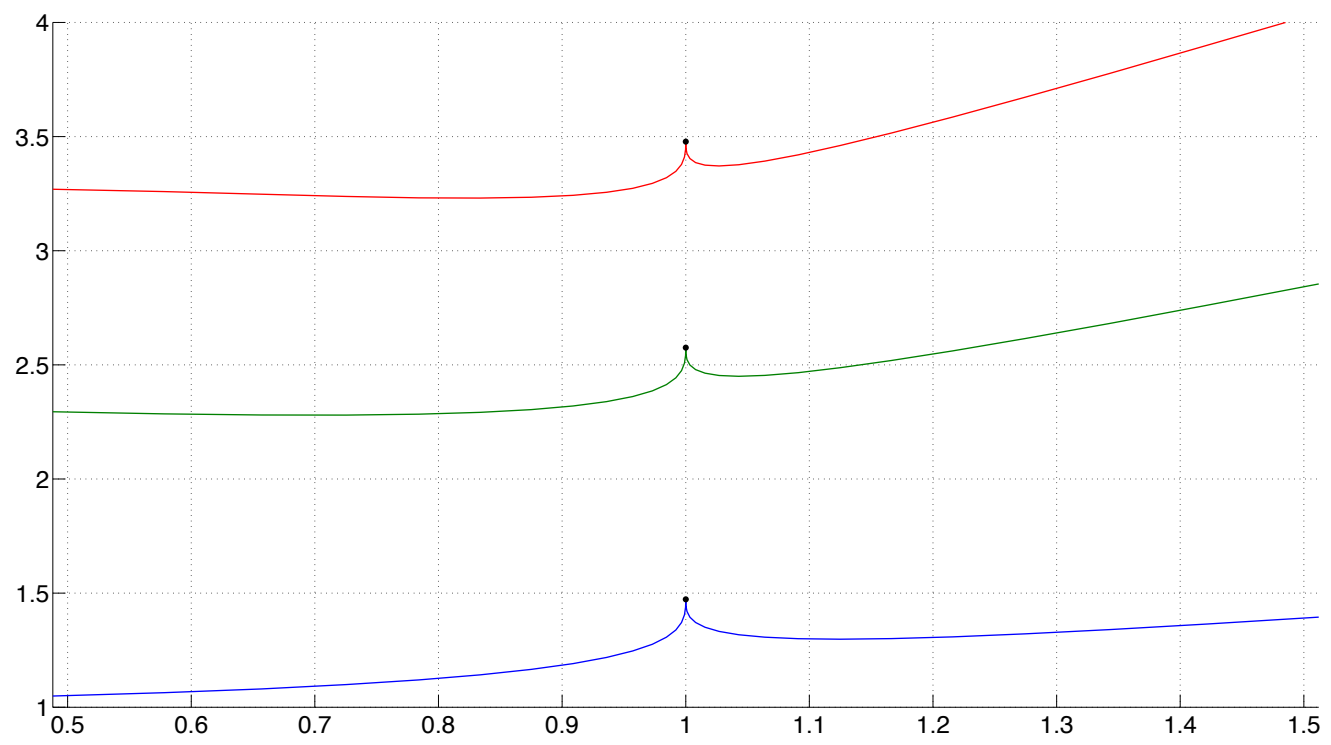

Figure 3: This figure represents the dependence of $\kappa_{n}(s)$ on $s$ for $n=1,2,3$. The black dots represent the values $2^{-2 / 3} z_{\mathrm{A}}(n)$ for $n=1,2,3$.

\section{Proof of the main theorem}

The aim of this section is to prove Theorem 1.2. We first perform the following change of variables to transfer the dependence on $h$ of $-\Delta_{\widehat{T r i}(s, h)}$ into the coefficient of the operator:

$$
x=x_{1}-s ; \quad y=\frac{1}{h} x_{2} .
$$

The triangle $\widehat{\operatorname{Tri}}(s, h)$ is transformed into a triangle $\operatorname{Tri}(s)$, which simply is a translation of $\widehat{\operatorname{Tri}}(s, 1)$. The operator $-h^{2} \Delta_{\widehat{T r i}(s, h)}^{\text {Dir }}$ becomes

$$
\mathcal{L}_{s}(h):=-h^{2} \partial_{x}^{2}-\partial_{y}^{2} .
$$

It also has compact resolvent and we denote by $\left(\lambda_{n}(s, h)\right)_{n \geq 1}$ its eigenvalues. They satisfy

$$
\lambda_{n}(s, h)=\mu_{n}(s, h) h^{2} .
$$

The operator $\mathcal{L}_{s}(h)$ defined in (3.2) is partially semiclassical in $x$. Its investigation follows the lines of the papers $[9,12,13]$.

The proof is divided into two main steps: a construction of quasimodes and the use of the true eigenfunctions of $\mathcal{L}_{s}(h)$ as quasimodes for the model operator in order to obtain a lower bound for the true eigenvalues.

We first perform a change of variables to transform the triangle Tri $(s)$ into the rectangle $\operatorname{Rec}(s)=(-1-s, 1-s) \times(0,1):$

$$
\begin{cases}u=x ; & t=(1+s) \frac{y}{x+1+s} \quad \text { for }-1-s<x<0 \\ u=x ; \quad t=-(1-s) \frac{y}{x-(1-s)} & \text { for } 0<x<1-s .\end{cases}
$$


For the sake of simplicity we define

$$
s_{-}:=1+s, \quad s_{+}:=s-1 .
$$

In the coordinates $(u, t)$ defined in (3.4), we denote by $\mathcal{L}_{s}^{-}(h)$ and $\mathcal{L}_{s}^{+}(h)$ the expression of $\mathcal{L}_{s}(h)$, respectively for $u<0$ and $u>0$. We have:

$$
\mathcal{L}_{s}^{ \pm}(h)\left(u, t ; \partial_{u}, \partial_{t}\right):=-h^{2}\left(\partial_{u}^{2}-\frac{2 t}{u+s_{ \pm}} \partial_{u} \partial_{t}+\frac{2 t}{\left(u+s_{ \pm}\right)^{2}} \partial_{t}+\frac{t^{2}}{\left(u+s_{ \pm}\right)^{2}} \partial_{t}^{2}\right)-\frac{s_{ \pm}^{2}}{\left(u+s_{ \pm}\right)^{2}} \partial_{t}^{2} .
$$

To give a description of the domain of $\mathcal{L}_{s}^{ \pm}$we recall that

$$
\operatorname{Dom}\left(\mathcal{L}_{s}(h)\right)=H^{2}(\operatorname{Tri}(s)) \cap H_{0}^{1}(\operatorname{Tri}(s))
$$

$\operatorname{Dom}\left(\mathcal{L}_{s}(h)\right)$ can also be described as:

$$
\left\{\left(\Psi^{-}, \Psi^{+}\right) \in H^{2}\left(\operatorname{Tri}(s)^{-}\right) \times H^{2}\left(\operatorname{Tri}(s)^{+}\right):\left\{\begin{array}{l}
\Psi^{-}+\Psi^{+} \in H_{0}^{1}(\operatorname{Tri}(s)) \\
\Psi^{-}(0, y)=\Psi^{+}(0, y), \text { for all } y \in(0,1) \\
\partial_{x} \Psi^{-}(0, y)=\partial_{x} \Psi^{+}(0, y), \text { for all } y \in(0,1)
\end{array}\right\},\right.
$$

where $\operatorname{Tri}(s)^{ \pm}=\operatorname{Tri}(s) \cap\left\{(x, y) \in \mathbb{R}^{2}: x \in \mathbb{R}^{ \pm}\right\}$. The domain of $\mathcal{L}_{s}^{ \pm}(h)$ is obtained thanks to the change of variables (3.4). Particularly, the boundary conditions are Dirichlet on $(-1,1) \times\{0\}$ and $(-1,1) \times\{1\}$ and the transmission conditions become, for all $t \in(0,1)$ and $\psi^{ \pm} \in \operatorname{Dom}\left(\mathcal{L}_{s}^{ \pm}(h)\right)$ :

$$
\psi^{-}(0, t)=\psi^{+}(0, t) \quad \text { and } \quad\left(\partial_{u}-\frac{t}{s_{-}} \partial_{t}\right) \psi^{-}(0, t)=\left(\partial_{u}-\frac{t}{s_{+}} \partial_{t}\right) \psi^{+}(0, t),
$$

where $\psi^{ \pm}(u, t)=\Psi^{ \pm}\left(u, \frac{u+s_{ \pm}}{s_{ \pm}} t\right)$.

\subsection{Quasimodes}

To prove Theorem 1.2 we first construct quasimodes at any order in power of $h^{1 / 3}$ and it yields the

Proposition 3.1 Let $\mathfrak{S}\left(\mathcal{L}_{s}(h)\right)$ denotes the spectrum of $\mathcal{L}_{s}(h)$ and $s_{0} \in[0,1)$. There are sequences $\left(\beta_{j, n}(s)\right)_{j \geq 0}$ for any integer $n \geq 1$ so that there holds: for all $N_{0} \in \mathbb{N}^{*}$ and $J \in \mathbb{N}$, there exist $h_{0}>0$ and $C>0$ such that for all $s \in\left[-s_{0}, s_{0}\right]$ and all $h \in\left(0, h_{0}\right)$

$$
\operatorname{dist}\left(\mathfrak{S}\left(\mathcal{L}_{s}(h)\right), \sum_{j=0}^{J} \beta_{j, n}(s) h^{j / 3}\right) \leq C h^{(J+1) / 3}, \quad n=1, \ldots, N_{0} .
$$

Moreover, the functions $\left(s \mapsto \beta_{j, n}(s)\right)$ are analytic on $(-1,1)$ and we have: $\beta_{0, n}(s)=\pi^{2}, \beta_{1, n}(s)=0$, and $\beta_{2, n}(s)=\left(2 \pi^{2}\right)^{2 / 3} \kappa_{n}(s)$.

Proof: The proof is divided into three parts. The first one deals with the form of the Ansatz chosen to construct quasimodes. The second part deals with lemmas about operators which appear in the first part. The third part is the determination of the profiles of the Ansatz. 
Ansatz We want to construct quasimodes $\left(\gamma_{s, h}, \psi_{s, h}\right)$ for the operator $\mathcal{L}_{s}(h)\left(x, y ; \partial_{x}, \partial_{y}\right)$. It will be more convenient to work in the rectangle $\operatorname{Rec}(s)$ with the operators $\mathcal{L}_{s}^{ \pm}(h)\left(u, t ; \partial_{u}, \partial_{t}\right)$. We introduce the new scales:

$$
\alpha=h^{-2 / 3} u ; \quad \beta=h^{-1} u .
$$

We look for quasimodes $\hat{\psi}_{s, h}(u, t)=\psi_{s, h}(x, y)$. Such quasimodes will have the form on the left and on the right:

$$
\psi_{s}^{ \pm}(u, t) \sim \sum_{j \geq 0}\left[\Psi_{s, j}^{ \pm}(\alpha, t)+\Phi_{s, j}^{ \pm}(\beta, t)\right] h^{j / 3}
$$

associated with quasi-eigenvalues:

$$
\gamma_{s, h} \sim \sum_{j \geq 0} \beta_{j}(s) h^{j / 3}
$$

in order to solve the eigenvalue equation in the sense of formal series. An Ansatz containing the scale $h^{2 / 3}$ alone is not sufficient to construct quasimodes because one can see that the system is overdetermined. Expanding the operators in powers of $h^{2 / 3}$, we obtain the formal series:

$\mathcal{L}_{s}^{ \pm}(h)\left(h^{2 / 3} \alpha, t ; h^{-2 / 3} \partial_{\alpha}, \partial_{t}\right) \sim \sum_{j \geq 0} \mathcal{L}_{s, 2 j}^{ \pm}\left(\alpha, t ; \partial_{\alpha}, \partial_{t}\right) h^{2 j / 3}$, with leading terms $\left\{\begin{array}{l}\mathcal{L}_{s, 0}^{ \pm}:=\mathcal{L}_{0}^{ \pm}=-\partial_{t}^{2} \\ \mathcal{L}_{s, 2}^{ \pm}:=\frac{2 \alpha}{s_{ \pm}} \partial_{t}^{2}-\partial_{\alpha}^{2}\end{array}\right.$,

and in power of $h$ :

$\mathcal{L}_{s}^{ \pm}(h)\left(h \beta, t ; h^{-1} \partial_{\beta}, \partial_{t}\right) \sim \sum_{j \geq 0} \mathcal{N}_{s, 3 j}^{ \pm}\left(\beta, t ; \partial_{\beta}, \partial_{t}\right) h^{j}$, with leading terms $\left\{\begin{array}{l}\mathcal{N}_{s, 0}^{ \pm}:=\mathcal{N}_{0}^{ \pm}=-\partial_{\beta}^{2}-\partial_{t}^{2} \\ \mathcal{N}_{s, 3}^{ \pm}:=\frac{2 t}{s_{ \pm}} \partial_{\beta} \partial_{t}-\frac{2 \beta}{s_{ \pm}} \partial_{t}^{2}\end{array}\right.$.

We consider these operators on the half-strips $\mathrm{H}_{-}:=(-\infty, 0) \times(0,1)$ and $\mathrm{H}_{+}:=(0, \infty) \times(0,1)$. On the left and on the right, the leading term at the scale $h^{2 / 3}$ acts only in the transverse variable $t$ and is the Dirichlet Laplacian on $(0,1)$. At the scale $h$, the leading term is the Laplacian on a half-strip. The leading terms at both scales do not depend on $s$. Since $\psi_{s, h}$ has no jump across the line $x=0$, we find that $\psi_{s}^{-}$and $\psi_{s}^{+}$should statisfy the two transmission conditions (3.5) on the interface $I:=\{0\} \times(0,1)$. For the formal series, these conditions write for all $t \in(0,1)$ and all $j \geq 0$ :

$$
\begin{gathered}
\Psi_{s, j}^{-}(0, t)+\Phi_{s, j}^{-}(0, t)=\Psi_{s, j}^{+}(0, t)+\Phi_{s, j}^{+}(0, t) \\
\partial_{\alpha} \Psi_{s, j-1}^{-}(0, t)+\partial_{\beta} \Phi_{s, j}^{-}(0, t)-\frac{t}{s_{-}} \partial_{t} \Psi_{s, j-3}^{-}(0, t)-\frac{t}{s_{-}} \partial_{t} \Phi_{s, j-3}^{-}(0, t) \\
=\partial_{\alpha} \Psi_{s, j-1}^{+}(0, t)+\partial_{\beta} \Phi_{s, j}^{+}(0, t)-\frac{t}{s_{+}} \partial_{t} \Psi_{s, j-3}^{+}(0, t)-\frac{t}{s_{+}} \partial_{t} \Phi_{s, j-3}^{+}(0, t),
\end{gathered}
$$

where we understand that the terms associated with a negative index are 0 . Finally, in order to ensure the Dirichlet boundary condition on $\operatorname{Tri}(s)$ we will require for our Ansatz, for any $j \in \mathbb{N}$, the boundary conditions:

$$
\Psi_{s, j}^{ \pm}(\cdot, 0 \text { and } 1)=0 ; \quad \Phi_{s, j}^{ \pm}(\cdot, 0 \text { and } 1)=0
$$

Three lemmas To start the construction of our Ansatz, we will need three lemmas, but before let us denote by $\left(\mathfrak{s}_{j}\right)_{j \geq 1}$ the eigenfunctions associated with the eigenvalues of the Dirichlet Laplacian on the interval $(0,1)$. We have $\mathfrak{s}_{j}(t)=\sqrt{2} \sin (j \pi t)$ and this eigenfunction is associated with the eigenvalue $j^{2} \pi^{2}$.

The analyticity of the solutions in the following lemmas is a direct consequence of the analyticity of the datas. 
Lemma 3.2 Let $F_{s}^{-}=F_{s}^{-}(\beta, t)$ and $F_{s}^{+}=F_{s}^{+}(\beta, t)$ be functions respectively in $L_{\exp }^{2}\left(\mathrm{H}_{-}\right)$and $L_{\text {exp }}^{2}\left(\mathrm{H}_{+}\right)$, depending analytically on $s \in(-1,1)$. Let $G_{s} \in H^{3 / 2}(I) \cap H_{0}^{1}(I)$ and $H_{s} \in H^{1 / 2}(I)$ be data on the interface $I$, depending analytically of $s \in(-1,1)$. Then, for all $s \in(-1,1)$ there exist unique coefficients $\xi_{s}$ and $\delta_{s}$ such that the transmission problem:

$$
\left\{\begin{array}{l}
\left(\mathcal{N}_{0}^{ \pm}-\pi^{2}\right) \Phi_{s}^{ \pm}=F_{s}^{ \pm} \quad \text { in } \mathrm{H}_{ \pm}, \quad \Phi_{s}^{ \pm}(\cdot, 0 \text { and } 1)=0, \\
\Phi_{s}^{-}(0, t)-\Phi_{s}^{+}(0, t)=G_{s}(t)+\xi_{s} \mathfrak{s}_{1}(t) \\
\partial_{\beta} \Phi_{s}^{-}(0, t)-\partial_{\beta} \Phi_{s}^{+}(0, t)=H_{s}(t)+\delta_{s} \mathfrak{s}_{1}(t),
\end{array}\right.
$$

has a unique solution $\left(\Phi_{s}^{-}, \Phi_{s}^{+}\right)$in $H_{\exp }^{2}\left(\mathrm{H}_{-}\right) \times H_{\exp }^{2}\left(\mathrm{H}_{+}\right)$and we have

$$
\begin{gathered}
\xi_{s}=-\int_{-\infty}^{0}\left\langle F_{s}^{-}(\beta, \cdot), \mathfrak{s}_{1}\right\rangle_{t} \beta \mathrm{d} \beta-\int_{0}^{+\infty}\left\langle F_{s}^{+}(\beta, \cdot), \mathfrak{s}_{1}\right\rangle_{t} \beta \mathrm{d} \beta-\left\langle G_{s}, \mathfrak{s}_{1}\right\rangle_{t}, \\
\delta_{s}=\int_{-\infty}^{0}\left\langle F_{s}^{-}(\beta, \cdot), \mathfrak{s}_{1}\right\rangle_{t} \mathrm{~d} \beta-\int_{0}^{+\infty}\left\langle F_{s}^{+}(\beta, \cdot), \mathfrak{s}_{1}\right\rangle_{t} \mathrm{~d} \beta-\left\langle H_{s}, \mathfrak{s}_{1}\right\rangle_{t} .
\end{gathered}
$$

Moreover $\xi_{s}, G_{s}$ and $\left(\Phi_{s}^{-}, \Phi_{s}^{+}\right)$depend analytically on $s \in(-1,1)$.

Proof of the lemma: We look for a solution $\left(\Phi_{s}^{-}, \Phi_{s}^{+}\right)$that we decompose, in the transverse coordinates, along the basis of the eigenfunctions of the Dirichlet Laplacian on $(0,1)$ :

$$
\Phi_{s}^{ \pm}(\beta, t)=\sum_{j \geq 1} \Phi_{s, j}^{ \pm}(\beta) \mathfrak{s}_{j}(t)
$$

For all $j \geq 1$, the following equations are satisfied:

$$
\left(-\partial_{\beta}^{2}+\pi^{2}\left(j^{2}-1\right)\right) \Phi_{s, j}^{ \pm}=\left\langle F_{s}^{ \pm}, \mathfrak{s}_{j}\right\rangle_{t},
$$

and we are looking for exponentially decaying solutions. For $j=1$, we find:

$$
\Phi_{s, 1}^{-}(\beta)=\int_{-\infty}^{\beta} \int_{-\infty}^{\beta_{1}}\left\langle F_{s}^{-}\left(\beta_{2}, \cdot\right), \mathfrak{s}_{1}\right\rangle_{t} \mathrm{~d} \beta_{2} \mathrm{~d} \beta_{1}, \quad \Phi_{s, 1}^{+}(\beta)=-\int_{\beta}^{+\infty} \int_{\beta_{1}}^{+\infty}\left\langle F_{s}^{+}\left(\beta_{2}, \cdot\right), \mathfrak{s}_{1}\right\rangle_{t} \mathrm{~d} \beta_{2} \mathrm{~d} \beta_{1} .
$$

Using the data on the interface $I$ we find the expression of $\xi_{s}$ and $\delta_{s}$. For $j \geq 2$, we solve the ordinary differentials equations. It yields the existence of $A_{j}^{+}, B_{j}^{+}, A_{j}^{-}, B_{j}^{-} \in \mathbb{R}$, such that:

$$
\begin{aligned}
\Phi_{s, j}^{+}(\beta)= & A_{j}^{+} e^{\beta \pi \sqrt{j^{2}-1}}+B_{j}^{+} e^{-\beta \pi \sqrt{j^{2}-1}}+\frac{1}{2 \pi \sqrt{j^{2}-1}} e^{\beta \pi \sqrt{j^{2}-1}} \int_{\beta}^{+\infty} e^{-\beta_{1} \pi \sqrt{j^{2}-1}}\left\langle F_{s}^{+}(u, \cdot), \mathfrak{s}_{j}\right\rangle_{t} \mathrm{~d} \beta_{1} \\
& +\frac{1}{2 \pi \sqrt{j^{2}-1}} e^{-\beta \pi \sqrt{j^{2}-1}} \int_{\beta}^{+\infty} e^{\beta_{1} \pi \sqrt{j^{2}-1}}\left\langle F_{s}^{+}(u, \cdot), \mathfrak{s}_{j}\right\rangle_{t} \mathrm{~d} \beta_{1}
\end{aligned}
$$

and

$$
\begin{aligned}
\Phi_{s, j}^{-}(\beta)= & A_{j}^{-} e^{\beta \pi \sqrt{j^{2}-1}}+B_{j}^{-} e^{-\beta \pi \sqrt{j^{2}-1}}+\frac{1}{2 \pi \sqrt{j^{2}-1}} e^{\beta \pi \sqrt{j^{2}-1}} \int_{-\infty}^{\beta} e^{-\beta_{1} \pi \sqrt{j^{2}-1}}\left\langle F_{s}^{-}(u, \cdot), \mathfrak{s}_{j}\right\rangle_{t} \mathrm{~d} \beta_{1} \\
& +\frac{1}{2 \pi \sqrt{j^{2}-1}} e^{-\beta \pi \sqrt{j^{2}-1}} \int_{-\infty}^{\beta} e^{\beta_{1} \pi \sqrt{j^{2}-1}}\left\langle F_{s}^{-}(u, \cdot), \mathfrak{s}_{j}\right\rangle_{t} \mathrm{~d} \beta_{1},
\end{aligned}
$$

As we are looking for solutions in $H_{\text {exp }}^{2}\left(\mathrm{H}_{+}\right)$, we necessarily have $A_{j}^{+}=B_{j}^{-}=0 . B_{j}^{+}$and $A_{j}^{-}$are determined by the data on the interface $I$. It achieves the proof of Lemma 3.2.

The following lemma can be found in [7, Sec. 5]. It is a consequence of the Fredholm alternative: 
Lemma 3.3 Let $F_{s}^{ \pm}=F_{s}^{ \pm}(\alpha, t)$ be a function in $L_{\text {exp }}^{2}\left(\mathrm{H}_{ \pm}\right)$, and depending analytically on $s \in(-1,1)$. Then, there exist solution( $s) \Psi_{s}^{ \pm} \in H_{\exp }^{2}\left(\mathrm{H}_{ \pm}\right)$such that:

$$
\left(\mathcal{L}_{0}^{ \pm}-\pi^{2}\right) \Psi_{s}^{ \pm}=F_{s}^{ \pm} \quad \text { in } \mathrm{H}_{ \pm}, \quad \Psi_{s}^{ \pm}(\alpha, 0 \text { and } 1)=0
$$

if and only if:

$$
\left\langle F_{s}^{ \pm}(\alpha, \cdot), \mathfrak{s}_{1}\right\rangle_{t}=0 \text { for all } \alpha \in \mathbb{R}_{ \pm}^{*}
$$

In this case, they write:

$$
\Psi_{s}^{ \pm}(\alpha, t)=\Psi_{s}^{ \pm, \perp}(\alpha, t)+g_{s}^{ \pm}(\alpha) \mathfrak{s}_{1}(t)
$$

with $\Psi_{s}^{ \pm, \perp} \in H_{\exp }^{2}\left(\mathrm{H}_{ \pm}\right)$. Moreover, $\Psi_{s}^{ \pm}, \Psi_{s}^{ \pm, \perp}$ and $g_{s}^{ \pm}$are analytic in the s-variable.

Lemma 3.4 Let $f_{s}^{-}=f_{s}^{-}(\alpha) \in L_{\exp }^{2}\left(\mathbb{R}_{-}\right), f_{s}^{+}=f_{s}^{+}(\alpha) \in L_{\exp }^{2}\left(\mathbb{R}_{+}\right)$and $c_{s}, \theta_{s} \in \mathbb{R}$ depending analytically on $s \in(-1,1)$. If $\mathrm{T}_{s}^{n}$ is the function defined in Proposition 2.4, there exists a unique $\omega(s)$ such that the system

$$
\begin{cases}\left(-\partial_{\alpha}^{2}-\frac{\alpha}{1+s}-\kappa_{n}(s)\right) g_{s}^{-}=f_{s}^{-}+\omega(s) \mathrm{T}_{s}^{n} \quad \text { in } \mathbb{R}_{-}, & g_{s}^{+}(0)-g_{s}^{-}(0)=c_{s} \\ \left(-\partial_{\alpha}^{2}+\frac{\alpha}{1-s}-\kappa_{n}(s)\right) g_{s}^{+}=f_{s}^{+}+\omega(s) \mathrm{T}_{s}^{n} \quad \text { in } \mathbb{R}_{+}, & \left(g_{s}^{\text {rig }}\right)^{\prime}(0)-\left(g_{s}^{\text {lef }}\right)^{\prime}(0)=\theta_{s},\end{cases}
$$

has a unique solution $\left(g_{s}^{-}, g_{s}^{+}\right) \in H_{\exp }^{2}\left(\mathbb{R}_{-}\right) \times H_{\exp }^{2}\left(\mathbb{R}_{+}\right)$. Moreover $g_{s}^{-}, g_{s}^{+}$and $\omega(s)$ depend analytically on $s$.

Proof of the lemma: Let us define $g_{s}:=g_{s}^{-} \mathbb{1}_{\mathbb{R}_{-}}+g_{s}^{+} \mathbb{1}_{\mathbb{R}_{+}}$. In the distribution sense we have:

$$
\left(l_{s}^{\text {mod }}-\kappa_{n}(s)\right) g_{s}=\left(l_{s}^{\text {mod }}-\kappa_{n}(s)\right)\left(g_{s}^{-} \mathbb{1}_{\mathbb{R}_{-}}\right)+\left(l_{s}^{\text {mod }}-\kappa_{n}(s)\right)\left(g_{s}^{+} \mathbb{1}_{\mathbb{R}_{+}}\right) .
$$

After computations we find:

$$
\left(l_{s}^{\mathrm{mod}}-\kappa_{n}(s)\right) g_{s}=f_{s}+\omega(s) \mathrm{T}_{s}^{n}-\theta_{s} \delta_{0}-c_{s} \delta_{0}^{\prime}
$$

where $f_{s}:=f_{s}^{-} \mathbb{1}_{\mathbb{R}_{-}}+f_{s}^{+} \mathbb{1}_{\mathbb{R}_{+}}$and $\delta_{0}$ is the Dirac delta function at $\alpha=0$. Then, we define the function $m$ :

$$
m(\alpha):=\left(\theta_{s} \alpha+c_{s}\right) \mathbb{1}_{\mathbb{R}_{+}}
$$

In the distribution sense, we have:

$$
m^{\prime}(\alpha)=\theta_{s} \mathbb{1}_{\mathbb{R}_{+}}+c_{s} \delta_{0}, \quad m^{\prime \prime}(\alpha)=\theta_{s} \delta_{0}+c_{s} \delta_{0}^{\prime}
$$

We introduce a smooth cut-off function $\chi$ which is 1 near 0 . Finally, we define the auxiliary function $\tilde{g}_{s}:=g_{s}-\chi m$ and we obtain:

$$
\left(l_{s}^{\text {mod }}-\kappa_{n}(s)\right) \tilde{g}_{s}=f_{s}+\omega(s) \mathrm{T}_{s}^{n}+\left(\left(\partial_{\alpha}^{2} \chi\right) m+2 \theta_{s}\left(\partial_{\alpha} \chi\right) \mathbb{1}_{\mathbb{R}_{+}}-v_{s}^{\text {mod }}(\alpha) \chi m+\kappa_{n}(s) \chi m\right) .
$$

By definition, $\tilde{g}_{s}$ belongs to the form domain of the operator $l_{s}^{\text {mod }}$. The right hand side of equation (3.9) being in $L^{2}(\mathbb{R}), \tilde{g}_{s}$ is also in the domain of the operator $l_{s}^{\text {mod }}$. As a consequence, equation (3.9) is also true in $L^{2}(\mathbb{R})$ and we can apply the Fredholm alternative to find a solution $\tilde{g}_{s}$ and $\omega(s)$, this latest satisfying:

$$
\omega(s)=\left\langle\left(v_{s}^{\text {mod }}-\kappa_{n}(s)\right) \chi m-\left(\partial_{\alpha}^{2}\right) m-2 \theta_{s}\left(\partial_{\alpha} \chi\right) \mathbb{1}_{\mathbb{R}_{+}}-f_{s}, \mathrm{~T}_{s}^{n}\right\rangle .
$$

It concludes the proof of Lemma 3.4.

In the following construction we use a version of this lemma up to some normalization constants. 


\section{Determination of the profiles}

Now we can start the construction of the Ansatz.

Terms of order $h^{0} \quad$ Let us write the equations inside the strip:

$$
\mathrm{H}_{ \pm, \alpha}: \quad-\partial_{t}^{2} \Psi_{s, 0}^{ \pm}=\gamma_{0}(s) \Psi_{s, 0}^{ \pm} \quad \mathrm{H}_{ \pm, \beta}: \quad-\left(\partial_{t}^{2}+\partial_{\beta}^{2}\right) \Phi_{s, 0}^{ \pm}=\gamma_{0}(s) \Phi_{s, 0}^{ \pm}
$$

The transmission conditions are:

$$
\begin{gathered}
\left(\Psi_{s, 0}^{-}+\Phi_{s, 0}^{-}\right)(0, t)=\left(\Psi_{s, 0}^{+}+\Phi_{s, 0}^{+}\right)(0, t) \\
\left(\partial_{\beta} \Phi_{s, 0}^{-}-\partial_{\beta} \Phi_{s, 0}^{+}\right)(0, t)=0
\end{gathered}
$$

With the Dirichlet boundary conditions (3.8), we get:

$$
\gamma_{0}(s)=\pi^{2}, \quad \Psi_{s, 0}^{ \pm}(\alpha, t)=g_{s, 0}^{ \pm}(\alpha) \mathfrak{s}_{1}(t) .
$$

We now apply Lemma 3.2 with $F_{s}^{-} \equiv 0, F_{s}^{+} \equiv 0, G_{s} \equiv 0$ and $H_{s} \equiv 0$ to get:

$$
\xi_{s}=0 \quad \text { and } \quad \delta_{s}=0
$$

We deduce $\Phi_{s, 0}^{-} \equiv 0$ and $\Phi_{s, 0}^{+} \equiv 0$ and, since $\xi_{s}=g_{s, 0}^{+}(0)-g_{s, 0}^{-}(0), g_{s, 0}^{+}(0)=g_{s, 0}^{-}(0)$. At this step we do not have determined $g_{s, 0}^{ \pm}$yet.

Terms of order $h^{1 / 3}$ The equations inside the strip read:

$$
\mathrm{H}_{ \pm, \alpha}: \quad\left(-\partial_{t}^{2}-\pi^{2}\right) \Psi_{s, 1}^{ \pm}=\gamma_{1}(s) \Psi_{s, 0}^{ \pm} \quad \mathrm{H}_{ \pm, \beta}: \quad\left(-\partial_{t}^{2}-\partial_{\beta}^{2}-\pi^{2}\right) \Phi_{s, 1}^{ \pm}=0
$$

The transmission conditions are:

$$
\begin{aligned}
\left(\Phi_{s, 1}^{-}+\Psi_{s, 1}^{-}\right)(0, t) & =\left(\Phi_{s, 1}^{+}+\Psi_{s, 1}^{+}\right)(0, t) \\
\left(\partial_{\alpha} \Psi_{s, 0}^{-}+\partial_{\beta} \Phi_{s, 1}^{-}\right)(0, t) & =\left(\partial_{\alpha} \Psi_{s, 0}^{+}+\partial_{\beta} \Phi_{s, 1}^{+}\right)(0, t) .
\end{aligned}
$$

We also take the Dirichlet boundary conditions (3.8) into account. Lemma 3.3 implies:

$$
\gamma_{1}(s)=0, \quad \Psi_{s, 1}^{ \pm}(\alpha, t)=g_{s, 1}^{ \pm}(\alpha) \mathfrak{s}_{1}(t) .
$$

We now apply Lemma 3.2 with $F_{s}^{-} \equiv 0, F_{s}^{+} \equiv 0, G_{s} \equiv 0$ and $H_{s} \equiv 0$, we get:

$$
\xi_{s}=0, \quad \delta_{s}=0
$$

Since $\xi_{s}=g_{s, 1}^{+}(0)-g_{s, 1}^{-}(0)$ and $\delta_{s}=\left(g_{s, 0}^{+}\right)^{\prime}(0)-\left(g_{s, 0}^{-}\right)^{\prime}(0)$ we have:

$$
g_{s, 1}^{+}(0)=g_{s, 1}^{-}(0), \quad\left(g_{s, 0}^{+}\right)^{\prime}(0)=\left(g_{s, 0}^{-}\right)^{\prime}(0) .
$$

We also deduce that $\Phi_{s, 1}^{-} \equiv 0$ and $\Phi_{s, 1}^{+} \equiv 0$. 
Terms of order $h^{2 / 3}$ The equations inside the strip read:

$$
\mathrm{H}_{ \pm, \alpha}: \quad\left(-\partial_{t}^{2}-\pi^{2}\right) \Psi_{s, 2}^{ \pm}=-\mathcal{L}_{s, 2}^{ \pm} \Psi_{s, 0}^{ \pm}+\gamma_{2}(s) \Psi_{s, 0}^{ \pm} \quad \mathrm{H}_{ \pm, \beta}: \quad\left(-\partial_{t}^{2}-\partial_{\beta}^{2}-\pi^{2}\right) \Phi_{s, 2}^{ \pm}=0
$$

where we have:

$$
\mathcal{L}_{s, 2}^{ \pm}:=\frac{2 \alpha}{s_{ \pm}} \partial_{t}^{2}-\partial_{\alpha}^{2}
$$

The transmission conditions are:

$$
\begin{aligned}
\left(\Psi_{s, 2}^{-}+\Phi_{s, 2}^{-}\right)(0, t) & =\left(\Psi_{s, 2}^{+}+\Phi_{s, 2}^{+}\right)(0, t), \\
\partial_{\alpha} \Psi_{s, 1}^{-}(0, t)+\partial_{\beta} \Phi_{s, 2}^{-}(0, t) & =\partial_{\alpha} \Psi_{s, 1}^{+}(0, t)+\partial_{\beta} \Phi_{s, 2}^{+}(0, t),
\end{aligned}
$$

We also have to take the Dirichlet boundary conditions (3.8) into account. Then, we apply a renormalized version of Lemma 3.3. Consequently, there exists a solution $\left(\Psi_{s, 2}^{-}, \Psi_{s, 2}^{+}\right)$if and only if the following system is verified:

$$
\left\{\begin{array}{l}
\left(-\partial_{\alpha}^{2}-\frac{2 \alpha \pi^{2}}{1+s}-\gamma_{2}(s)\right) g_{s, 0}^{-}(\alpha)=0 \quad \text { in } \mathbb{R}_{-}, \quad g_{s, 0}^{+}(0)-g_{0}^{-}(0)=0 \\
\left(-\partial_{\alpha}^{2}+\frac{2 \alpha \pi^{2}}{1-s}-\gamma_{2}(s)\right) g_{s, 0}^{+}(\alpha)=0 \quad \text { in } \mathbb{R}_{+}, \quad\left(g_{s, 0}^{+}\right)^{\prime}(0)-\left(g_{s, 0}^{-}\right)^{\prime}(0)=0 .
\end{array}\right.
$$

This leads to the choice:

$$
\gamma_{2}(s)=\left(2 \pi^{2}\right)^{2 / 3} \kappa_{n}(s) ; \quad g_{s, 0}(\alpha)=\mathrm{T}_{s}^{n}\left(\left(2 \pi^{2}\right)^{2 / 3} \alpha\right),
$$

with $g_{s, 0}^{ \pm}(\alpha)=\mathrm{T}_{s}^{n}\left(\left(2 \pi^{2}\right)^{2 / 3} \alpha\right) \mathbb{1}_{\mathbb{R}_{ \pm}}(\alpha)$. Particularly, this determines the unknown functions of the previous steps (this choice of $g_{s, 0}$ gives an explicit expression of $\Psi_{s, 0}^{ \pm}$). We are led to take:

$$
\Psi_{s, 2}^{ \pm}(\alpha, t)=\Psi_{s, 2}^{ \pm, \perp}(\alpha, t)+g_{s, 2}^{ \pm}(\alpha) \mathfrak{s}_{1}(t)
$$

with $\Psi_{s, 2}^{+, \perp} \equiv 0$ and $\Psi_{s, 2}^{-, \perp} \equiv 0$. Finally we have to solve the system:

$$
\left\{\begin{array}{l}
\left(-\partial_{t}^{2}-\partial_{\beta}^{2}-\pi^{2}\right) \Phi_{s, 2}^{ \pm}=0 \text { in } \mathrm{H}_{ \pm} \\
\Phi_{s, 2}^{ \pm}(\cdot, 0 \text { and } 1)=0 \\
\Phi_{s, 2}^{-}(0, t)-\Phi_{s, 2}^{+}(0, t)=\left(g_{s, 2}^{+}(0)-g_{s, 2}^{-}(0)\right) \mathfrak{s}_{1}(t) \\
\partial_{\beta} \Phi_{s, 2}^{-}(0, t)-\partial_{\beta} \Phi_{s, 2}^{+}(0, t)=\left(\left(g_{s, 1}^{+}\right)^{\prime}(0)-\left(g_{s, 1}^{-}\right)^{\prime}(0)\right) \mathfrak{s}_{1}(t)
\end{array}\right.
$$

Then, we apply Lemma 3.2 with $F_{s}^{-} \equiv 0, F_{s}^{+} \equiv 0, G_{s} \equiv 0$ and $H_{s} \equiv 0=\left(\left(g_{s, 1}^{+}\right)^{\prime}(0)-\left(g_{s, 1}^{-}\right)^{\prime}(0)\right) \mathfrak{s}_{1}(t)$ and we get:

$$
\xi_{s}=g_{s, 2}^{+}(0)-g_{s, 2}^{-}(0)=0, \quad \delta_{s}=\left(g_{s, 1}^{+}\right)^{\prime}(0)-\left(g_{s, 1}^{-}\right)^{\prime}(0)=0 .
$$

This gives $\Phi_{s, 2}^{+} \equiv 0, \Phi_{s, 2}^{-} \equiv 0$.

Terms of order $h$ The equations inside the strip read:

$$
\mathrm{H}_{ \pm, \alpha}: \quad\left(-\partial_{t}^{2}-\pi^{2}\right) \Psi_{s, 3}^{ \pm}=\gamma_{3}(s) \Psi_{s, 0}^{ \pm} \quad \mathrm{H}_{ \pm, \beta}: \quad\left(-\partial_{t}^{2}-\partial_{\beta}^{2}-\pi^{2}\right) \Phi_{s, 3}^{ \pm}=0
$$

The transmission conditions are:

$$
\left(\Psi_{s, 3}^{-}+\Phi_{s, 3}^{-}\right)(0, t)=\left(\Psi_{s, 3}^{+}+\Phi_{s, 3}^{+}\right)(0, t)
$$




$$
\partial_{\alpha} \Psi_{s, 2}^{-}(0, t)+\partial_{\beta} \Phi_{s, 3}^{-}(0, t)-\frac{t}{s_{-}} \partial_{t} \Psi_{s, 0}^{-}(0, t)=\partial_{\alpha} \Psi_{s, 2}^{+}(0, t)+\partial_{\beta} \Phi_{s, 3}^{+}(0, t)-\frac{t}{s_{+}} \partial_{t} \Psi_{s, 0}^{+}(0, t) .
$$

We also take the Dirichlet boundary conditions (3.8) into account. Lemma 3.3 gives:

$$
\gamma_{3}(s)=0, \quad \Psi_{s, 3}^{ \pm}(\alpha, t)=g_{s, 3}^{ \pm}(\alpha) \mathfrak{s}_{1}(t) .
$$

We have to solve the system:

$$
\left\{\begin{array}{l}
\left(-\partial_{t}^{2}-\partial_{\beta}^{2}-\pi^{2}\right) \Phi_{s, 3}^{ \pm}=0 \quad \text { in } \mathrm{H}_{ \pm} \\
\Phi_{s, 3}^{ \pm}(\cdot, 0 \text { and } 1)=0 \\
\Phi_{s, 3}^{-}(0, t)-\Phi_{s, 3}^{+}(0, t)=\left(g_{s, 3}^{+}(0)-g_{s, 3}^{-}(0)\right) \mathfrak{s}_{1}(t) \\
\left(\partial_{\beta} \Phi_{s, 3}^{-}-\partial_{\beta} \Phi_{s, 3}^{+}\right)(0, t)=\left(\left(g_{s, 2}^{+}\right)^{\prime}(0)-\left(g_{s, 2}^{-}\right)^{\prime}(0)\right) \mathfrak{s}_{1}(t)+g_{s, 0}^{+}(0)\left(\frac{t \pi}{1-s}+\frac{t \pi}{1+s}\right) \cos (\pi t) .
\end{array}\right.
$$

Hence, we apply Lemma 3.2 with $F_{s}^{-} \equiv 0, F_{s}^{+} \equiv 0, G_{s}^{0} \equiv 0$ and $H_{s}(t)=g_{s, 0}^{+}(0)\left(\frac{t \pi}{1-s}+\frac{t \pi}{1+s}\right) \cos (\pi t)$ and we get:

$$
\xi_{s}=g_{s, 3}^{+}(0)-g_{s, 3}^{-}(0)=0, \quad \delta_{s}=\left(g_{s, 2}^{+}\right)^{\prime}(0)-\left(g_{s, 2}^{-}\right)^{\prime}(0)=-\frac{2 \sqrt{2} \pi}{1-s^{2}} \mathrm{~T}_{s}^{n}(0)\left\langle t \cos (\pi t), \mathfrak{s}_{1}\right\rangle_{t} .
$$

This determines $\Phi_{s, 3}^{-}$and $\Phi_{s, 3}^{+}$which are not necessarily zero.

Continuation Let us assume that we can write $\Psi_{s, k}^{ \pm}(\alpha, t)=\Psi_{s, k}^{ \pm, \perp}(\alpha, t)+g_{s, k}^{ \pm}(\alpha) \mathfrak{s}_{1}(t)$ for all $0 \leq k \leq n$ and that $\left(g_{s, k}^{ \pm}\right)_{0 \leq k \leq n-3},\left(\Psi_{s, k}^{ \pm, \perp}\right)_{0 \leq k \leq n-1}$ are determined. Let us also assume that $g_{s, n-2}^{-}(0)-g_{s, n-2}^{+}(0),\left(g_{s, n-2}^{-}\right)^{\prime}(0)-\left(g_{s, n-2}^{+}\right)^{\prime}(0),\left(\gamma_{k}(s)\right)_{0 \leq k \leq n}$ and $\left(\Phi_{s, k}^{ \pm}\right)_{0 \leq k \leq n-2}$ are already known. Finally, we assume that $g_{s, n-1}^{-}(0)-g_{s, n-1}^{+}(0), \Phi_{s, n-1}^{ \pm}$are known once $g_{s, n-2}^{-}$and $g_{s, n-2}^{+}$are determined and all these functions are exponentially decaying and analytic in the $s$ variable for $s \in(-1,1)$. The equations inside the strip read:

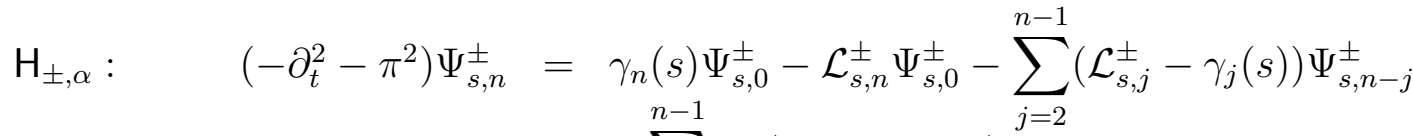

$$
\begin{aligned}
& \mathrm{H}_{ \pm, \beta}: \quad\left(-\partial_{t}^{2}-\partial_{\gamma}^{2}-\pi^{2}\right) \Phi_{s, n}^{ \pm}=-\sum_{j=1}^{n-1}\left(\mathcal{N}_{s, j}^{ \pm}-\gamma_{j}(s)\right) \Phi_{s, n-j}^{ \pm}{ }_{j=2}
\end{aligned}
$$

The transmission conditions are:

$$
\begin{aligned}
& \left(\Psi_{s, n}^{-}+\Phi_{s, n}^{-}(0, t)=\left(\Psi_{s, n}^{+}+\Phi_{s, n}^{+}\right)(0, t)\right. \\
\left(\partial_{\beta} \Phi_{s, n}^{-}-\partial_{\beta} \Phi_{s, n}^{+}\right)(0, t)= & \left(\left(g_{s, n-1}^{+}\right)^{\prime}(0)-\left(g_{s, n-1}^{-}\right)^{\prime}(0)\right) \mathfrak{s}_{1}(t)+\left(\partial_{\alpha} \Psi_{s, n-1}^{+, \perp}-\partial_{\alpha} \Psi_{s, n-1}^{-, \perp}\right)(0, t) \\
& +\frac{t}{1+s}\left(\partial_{t} \Psi_{s, n-3}^{-, \perp}+\partial_{t} \Phi_{s, n-3}^{-}\right)(0, t)+\frac{t}{1-s}\left(\partial_{t} \Psi_{s, n-3}^{+, \perp}+\partial_{t} \Phi_{s, n-3}^{+}\right)(0, t) \\
& +\sqrt{2} \pi\left(\frac{1}{1+s} g_{s, n-3}^{-}(0)+\frac{1}{1-s} g_{s, n-3}^{+}(0)\right) t \cos (\pi t) .
\end{aligned}
$$

In order to apply Lemma 3.3 we need to solve equations in the form:

$$
\left(-\partial_{\alpha}^{2}-\frac{2 \alpha}{1+s} \pi^{2}-\gamma_{2}(s)\right) g_{s, n-2}^{-}(\alpha)=\gamma_{n}(s) g_{s, 0}^{-}(\alpha)+f_{s}^{-}(\alpha)
$$




$$
\left(-\partial_{\alpha}^{2}+\frac{2 \alpha}{1-s} \pi^{2}-\gamma_{2}(s)\right) g_{s, n-2}^{+}(\alpha)=\gamma_{n}(s) g_{s, 0}^{+}(\alpha)+f_{s}^{+}(\alpha)
$$

We recall that $\gamma_{2}(s)$ is a rescaled version of $\kappa_{n}(s)$, consequently, we can apply Lemma 3.4 because $f_{s}^{ \pm}$, $g_{s, n-2}^{-}(0)-g_{s, n-2}^{+}(0)$ and $\left(g_{s, n-2}^{-}\right)^{\prime}(0)-\left(g_{s, n-2}^{+}\right)^{\prime}(0)$ are known. It provides an unique $\gamma_{n}(s)$, moreover $g_{s, n-2}^{ \pm}$are now determined. From the recursion assumption, we deduce that $\Phi_{s, n-1}^{ \pm}$are now determined. Lemma 3.3 uniquely determines $\Psi_{s, n}^{ \pm, \perp}$ such that:

$$
\Psi_{s, n}^{ \pm}(\alpha, t)=\Psi_{s, n}^{ \pm, \perp}(\alpha, t)+g_{s, n}^{ \pm}(\alpha) \mathfrak{s}_{1}(t) .
$$

We can now write the system in the form:

$$
\left\{\begin{array}{l}
\left(\mathcal{N}_{s, 0}^{ \pm}-\pi^{2}\right) \Phi_{s, n}^{ \pm}=F_{s}^{ \pm}, \text {in } \mathrm{H}_{ \pm} \\
\Phi_{s, n}^{ \pm}(\cdot, 0 \text { and } 1)=0 \\
\Phi_{s, n}^{-}(0, t)-\Phi_{s, n}^{+}(0, t)=\left(\Psi_{s, n}^{+, \perp}-\Psi_{s, n}^{-, \perp}\right)(0, t)+\left(g_{s, n}^{-}(0)-g_{s, n}^{+}(0)\right) \mathfrak{s}_{1}(t) \\
\partial_{\beta} \Phi_{s, n}^{-}(0, t)-\partial_{\beta} \Phi_{s, n}^{+}(0, t)=H_{s}(t)+\left(\left(g_{s, n-1}^{+}\right)^{\prime}(0)-\left(g_{s, n-1}^{-}\right)^{\prime}(0)\right) \mathfrak{s}_{1}(t)
\end{array}\right.
$$

where $H_{s}$ is known. We can apply Lemma 3.2 which determines $g_{s, n}^{-}(0)-g_{s, n}^{+}(0),\left(g_{s, n-1}^{+}\right)^{\prime}(0)-\left(g_{s, n-1}^{-}\right)^{\prime}(0)$, $\Phi_{s, n}^{-}$and $\Phi_{s, n}^{+}$.

Quasimodes The previous construction leads to introduce:

$$
\widehat{\psi}_{s, h}^{[J]}(u, t):=\sum_{j=0}^{J+2}\left(\Psi_{s, j}^{ \pm}\left(u h^{-2 / 3}, t\right)+\Phi_{s, j}^{ \pm}\left(u h^{-1}, t\right)\right) h^{j / 3}-u \chi^{ \pm}\left(u h^{-1}\right) R_{J, s, h}^{ \pm}(p), \text { when } u \in \mathbb{R}_{ \pm} .
$$

where the correctors are

$$
R_{J, s, h}^{ \pm}(t)=\partial_{\alpha} \Psi_{s, J+2}^{ \pm}(0, t) h^{J / 3}-\frac{t}{s_{ \pm}} \sum_{j=J}^{J+2}\left(\partial_{t} \Psi_{s, j}^{ \pm}(0, t)+\partial_{t} \Phi_{s, j}^{ \pm}(0, t)\right)
$$

are added to make $\widehat{\psi}_{s, h}^{[J]}$ satisfy the second transmission condition. Here $\chi^{ \pm}$are two smooth cut-off functions being 1 near 0 . Then, $\psi_{s, h}^{[J]}$ defined by

$$
\psi_{s, h}^{[J]}(x, y)=\chi(u) \widehat{\psi}_{s, h}^{[J]}(u, t)
$$

belongs to the domain of $\mathcal{L}_{s}(h)$ : By construction, $\widehat{\psi}_{s, h}^{[J]}$ belongs to $H^{2}(\mathbb{R} \times(0,1))$, the cut-off function insures that $\psi_{s, h}^{[J]}$ is supported on the triangle Tri $(s)$ and that the change of variables (3.4) transmits the regularity of $\widehat{\psi}_{s, h}^{[J]}$ to $\psi_{s, h}^{[J]}$. The transmission conditions being satisfied, it yields $\psi_{s, h}^{[J]} \in H^{2}(\operatorname{Tri}(s))$. Moreover, by construction, $\psi_{s, h}^{[J]}$ satisfies the Dirichlet boundary conditions on the boundary of Tri $(s)$.

Using the exponential decay, for all $s_{0} \in(-1,1), J \in \mathbb{N}$ we get the existence of $h_{0}>0$, $C\left(J, s_{0}, h_{0}\right)>0$ such that for all $s \in\left[-s_{0}, s_{0}\right]$ and all $h \in\left(0, h_{0}\right)$ :

$$
\left\|\left(\mathcal{L}_{s}(h)-\sum_{j=0}^{J} \gamma_{j}(s) h^{j / 3}\right) \psi_{s, h}^{[J]}\right\| \leq C\left(J, s_{0}, h_{0}\right) h^{(J+1) / 3} .
$$

Particularly, from Proposition 3.1, we deduce the

Corollary 3.5 Let $s_{0} \in[0,1)$. For all $N_{0} \in \mathbb{N}^{*}$, there exist $h_{0}>0$ and $C>0$ such that for all $s \in\left[-s_{0}, s_{0}\right]$ and all $h \in\left(0, h_{0}\right)$ we have

$$
\lambda_{N_{0}}(s, h) \leq \pi^{2}+\left(2 \pi^{2}\right)^{2 / 3} \kappa_{N_{0}}(s) h^{2 / 3}+C h^{4 / 3} .
$$




\subsection{Agmon estimates}

In order to prove Theorem 1.2, we need Agmon localization estimates about $\mathcal{L}_{s}(h)$ (see the work [2, 3] and in the semiclassical context [8, Chap. 6]) and [13]). We remark that thanks to Propositions 1.1 and 3.1 , for all $s_{0} \in(0,1)$ the $N_{0}$ lowest eigenvalues $\lambda_{s}$ of $\mathcal{L}_{s}(h)$ satisfy for all $s \in\left[-s_{0}, s_{0}\right]$ :

$$
\left|\lambda_{s}-\pi^{2}\right| \leq \Gamma_{0} h^{2 / 3}
$$

for some positive constant $\Gamma_{0}$ depending on $N_{0}$ and $s_{0}$. We define $\operatorname{Tri}^{ \pm}(s):=\operatorname{Tri}(s) \cap\left\{x \in \mathbb{R}_{ \pm}\right\}$. We have the following two Agmon localization estimates for the true eigenfunctions of $\mathcal{L}_{s}(h)$ :

Proposition 3.6 Let $s_{0} \in[0,1)$. Let $\Gamma_{0}>0$ and $\rho_{0} \in(0, \pi)$. There exist $h_{0}>0, C_{0}>0, \eta_{0}>0$ and $D_{ \pm}>0$ such that for all $s \in\left[-s_{0}, s_{0}\right], h \in\left(0, h_{0}\right)$ and all eigenpair $\left(\lambda_{s}, \psi_{s}\right)$ of $\mathcal{L}_{s}(h)$ satisfying $\left|\lambda_{s}-\pi^{2}\right| \leq \Gamma_{0} h^{2 / 3}$, we have:

$\int_{\operatorname{Tri}^{ \pm}(s)} e^{\Phi_{1}^{ \pm}(x) / h}\left(\left|\psi_{s}\right|^{2}+\left|h^{2 / 3} \partial_{x} \psi_{s}\right|^{2}\right) \mathrm{d} x \mathrm{~d} y \leq C_{0}\left\|\psi_{s}\right\|^{2}$ and $\int_{\operatorname{Tri}^{ \pm}(s)} e^{\Phi_{2}^{ \pm}(x) / h}\left(\left|\psi_{s}\right|^{2}+\left|h \partial_{x} \psi_{s}\right|^{2}\right) \mathrm{d} x \mathrm{~d} y \leq C_{0}\left\|\psi_{s}\right\|^{2}$,

with

$$
\Phi_{1}^{ \pm}(x):=\frac{\eta_{0}}{\sqrt{\left|s_{ \pm}\right|}}|x|^{3 / 2} \text { and } \Phi_{2}^{ \pm}(x):=-\rho_{0}\left|s_{ \pm}\right| \ln \left(D_{ \pm}^{-1}\left(x+s_{ \pm}\right)\right)
$$

In the regime $h \rightarrow 0$, Proposition 3.6 localizes the eigenfunctions of $\mathcal{L}_{s}(h)$ in a neighborhood of $\operatorname{Tri}(s) \cap\{x=0\}$ and gives decay estimates away from this set. These estimates justify that the Feshbach-Grushin projections of the true eigenfunctions of $\mathcal{L}_{s}(h)$ are good quasimodes for the one dimensional operator $\left(u \mapsto h^{2} l_{s}^{\tan }\left(u+s ; \partial_{u}\right)\right)$, see (1.2), that is the tangent approximation of the Born-Oppenheimer approximation of $\mathcal{L}_{s}(h)$.

Proof: Because the shape of the effective potential $v_{s}$ defined in (1.4) is close to the one of [7], the proof of Proposition 3.6 uses the technical background of Propositions 5.5. and 5.6 of [7]. First, we remark that for all $\psi \in \operatorname{Dom}\left(Q_{\operatorname{Tri}(s), h}\right)$ we have:

$$
\int_{\operatorname{Tr}(s)} h^{2}\left|\partial_{x} \psi\right|^{2}+\left|\partial_{y} \psi\right|^{2} \mathrm{~d} x \mathrm{~d} y \geq \int_{\operatorname{Tri}(s)} h^{2}\left|\partial_{x} \psi\right|^{2}+v_{s}(x-s)|\psi|^{2} \mathrm{~d} x \mathrm{~d} y,
$$

where $Q_{\operatorname{Tr}(s), h}$ is the quadratic form associated with $\mathcal{L}_{s}(h)$. Thanks to (3.12), in order to prove Proposition (3.6) it is enough to prove the Agmon estimates for the eigenpairs of the one dimensional operator derived from (1.1) through the change of variables (3.1)

$$
l_{s}(h)=-h^{2} \partial_{x}+v_{s}(x-s),
$$

with domain $\operatorname{Dom}\left(l_{s}(h)\right)=H^{2}(-1-s, 1-s) \cap H_{0}^{1}(-1-s, 1-s) \cap L^{2}\left(-1-s, 1-s ; v_{s}(x-s) \mathrm{d} x\right)$. Let $\left(\lambda_{s}, \psi_{s}\right)$ be an eigenpair of $l_{s}(h)$ such that $\left|\lambda_{s}-\pi\right| \leq \Gamma_{0} h^{2 / 3}$. For $\Phi$ a Lipshitz function we have the IMS formula (see [12]):

$$
\int_{-1-s}^{1-s} h^{2}\left|\partial_{x}\left(e^{\Phi / h} \psi_{s}\right)\right|^{2}+v_{s}(x-s)\left|e^{\Phi / h} \psi_{s}\right|^{2}-\left|\Phi^{\prime} e^{\Phi} \psi_{s}\right|^{2}-\lambda_{s}\left|e^{\Phi / h} \psi_{s}\right|^{2} \mathrm{~d} x=0 .
$$

We first prove the inequality near $x=0$. The convexity of $v_{s}$ combined with (3.13) yields:

$$
\int_{-1-s}^{1-s} h^{2}\left|\partial_{x}\left(e^{\Phi / h} \psi_{s}\right)\right|^{2} \mathrm{~d} x+\sum_{j= \pm} \int_{\mathrm{I}_{j}}\left(\pi^{2}+\frac{2 \pi^{2}}{\left|s_{j}\right|}|x|-\Phi^{\prime}(x)^{2}-\lambda_{s}\right)\left|e^{\Phi} \psi_{s}\right|^{2} \mathrm{~d} x \leq 0,
$$


where $I_{ \pm}=(-1-s, 1-s) \cap \mathbb{R}_{ \pm}$. We have:

$$
\int_{-1-s}^{1-s} h^{2}\left|\partial_{x}\left(e^{\Phi / h} \psi_{s}\right)\right|^{2} \mathrm{~d} x+\sum_{j= \pm} \int_{\mathrm{I}_{j}}\left(\frac{2 \pi^{2}}{\left|s_{j}\right|}|x|-\Phi^{\prime}(x)^{2}-\Gamma_{0} h^{2 / 3}\right)\left|e^{\Phi_{s}} \psi_{s}\right|^{2} \mathrm{~d} x \leq 0 .
$$

We are lead to take

$$
\Phi_{1}^{ \pm}(x)=\eta_{ \pm} \frac{1}{\sqrt{\left|s_{ \pm}\right|}}|x|^{3 / 2}
$$

with $\eta_{ \pm}$small enough. We define $\Phi_{1}(x)=\sum_{j= \pm} \Phi_{1}^{j}(x) \mathbb{1}_{I_{j}}$ and we get:

$$
\int_{-1-s}^{1-s} h^{2}\left|\partial_{x}\left(e^{\Phi_{1} / h} \psi_{s}\right)\right|^{2} \mathrm{~d} x+\sum_{j= \pm} \int_{\mathbf{l}_{j}}\left(\left(2 \pi^{2}-\frac{9}{4} \eta_{j}^{2}\right) \frac{|x|}{\left|s_{j}\right|}-\Gamma_{0} h^{2 / 3}\right)\left|e^{\Phi_{1}^{j} / h} \psi_{s}\right|^{2} \mathrm{~d} x \leq 0 .
$$

For $\eta_{ \pm}$small enough, there exists $\tilde{\eta}_{ \pm}>0$ such that:

$$
\int_{-1-s}^{1-s} h^{2}\left|\partial_{x}\left(e^{\Phi_{1} / h} \psi_{s}\right)\right|^{2} \mathrm{~d} x+\sum_{j= \pm} \int_{\mathrm{I}_{j}}\left(\frac{\tilde{\eta}_{j}}{\left|s_{j}\right|}|x|-\Gamma_{0} h^{2 / 3}\right)\left|e^{\Phi_{1}^{j} / h} \psi_{s}\right|^{2} \mathrm{~d} x \leq 0 .
$$

Let $\varepsilon>0$, we define

$$
I_{ \pm}^{1}=\left\{x \in \mathrm{I}_{ \pm}: \frac{\tilde{\eta}_{ \pm}}{\left|s_{ \pm}\right|}|x|-\Gamma_{0} h^{2 / 3} \geq \varepsilon h^{2 / 3}\right\}, \quad I_{ \pm}^{2}=\left\{x \in \mathrm{I}_{ \pm}: \frac{\tilde{\eta}_{ \pm}}{\left|s_{ \pm}\right|}|x|-\Gamma_{0} h^{2 / 3} \leq \varepsilon h^{2 / 3}\right\}
$$

Splitting the integral we obtain:

$$
\int_{-1-s}^{1-s} h^{2}\left|\partial_{x}\left(e^{\Phi_{1} / h} \psi_{s}\right)\right|^{2} \mathrm{~d} x+\varepsilon h^{2 / 3} \sum_{j= \pm} \int_{I_{j}^{1}}\left|e^{\Phi_{1}^{j} / h} \psi_{s}\right|^{2} \mathrm{~d} x \leq \Gamma_{0} h^{2 / 3} \sum_{j= \pm} \int_{I_{j}^{2}}\left|e^{\Phi_{1}^{j} / h} \psi_{s}\right|^{2} \mathrm{~d} x
$$

Finally, we have:

$$
\int_{-1-s}^{1-s} h^{2}\left|\partial_{x}\left(e^{\Phi_{1} / h} \psi_{s}\right)\right|^{2} \mathrm{~d} x+\varepsilon h^{2 / 3} \sum_{j= \pm} \int_{I_{j}^{1}}\left|e^{\Phi_{1}^{j} / h} \psi_{s}\right|^{2} \mathrm{~d} x \leq\left(\Gamma_{0}+\varepsilon\right) h^{2 / 3} \sum_{j= \pm} \int_{I_{j}^{2}}\left|e^{\Phi_{1}^{j} / h} \psi_{s}\right|^{2} \mathrm{~d} x .
$$

For $x \in I_{ \pm}^{2}, \Phi_{1}^{ \pm}$is bounded. When $s \in\left[-s_{0}, s_{0}\right]$ this bound can be chosen uniformly in $s$. This achieves the proof of the first inequality.

For the second inequality, thanks to (3.13) we get:

$$
\int_{-1-s}^{1-s} h^{2}\left|\partial_{x}\left(e^{\Phi / h} \psi_{s}\right)\right|^{2} \mathrm{~d} x+\sum_{j= \pm} \int_{I_{j}}\left(\pi^{2} s_{j}^{2}\left(x+s_{j}\right)^{-2}-\pi^{2}-\Phi^{\prime}(x)^{2}-\Gamma_{0} h^{2 / 3}\right)\left|e^{\Phi / h} \psi_{s}\right|^{2} \mathrm{~d} x \leq 0 .
$$

We are lead to take

$$
\Phi_{2}^{ \pm}(x)=-\rho\left|s_{ \pm}\right| \ln \left(D_{ \pm}^{-1}\left(x+s_{ \pm}\right)\right),
$$

with $\rho \in(0, \pi)$ et $D_{ \pm}>0$ small enough such that

$$
\left|s_{ \pm}\right|^{2}\left(\pi^{2}-\rho^{2}\right) D_{ \pm}^{-2}-\pi^{2}>0
$$


We define $\Phi_{2}(x)=\sum_{j= \pm} \Phi_{2}^{j}(x) \mathbb{1}_{I_{j}}$ and we have

$$
\int_{-1-s}^{1-s} h^{2}\left|\partial_{x}\left(e^{\Phi_{2} / h} \psi_{s}\right)\right|^{2} \mathrm{~d} x+\sum_{j= \pm} \int_{I_{j}} s_{j}^{2}\left(\pi^{2}-\rho^{2}\right)\left(\left(x+s_{j}\right)^{-2}-\pi^{2}-\Gamma_{0} h^{2 / 3}\right)\left|e^{\Phi_{2}^{j} / h} \psi_{s}\right|^{2} \mathrm{~d} x \leq 0 .
$$

Let $h_{0}>0$ such that

$$
\left|s_{ \pm}\right|^{2}\left(\pi^{2}-\rho^{2}\right) D_{ \pm}^{-2}-\pi^{2}-\Gamma_{0} h_{0}^{2 / 3}>0
$$

we define

$$
\begin{gathered}
I_{-}^{1}=\left(-1-s,-1-s+D_{-}\right), \quad I_{+}^{1}=\left(1-s-D_{+}, 1-s\right), \\
I_{-}^{2}=\left(-1-s+D_{-}, 0\right), \quad I_{+}^{2}=\left(0,1-s-D_{+}\right) .
\end{gathered}
$$

Consequently, for all $h \in\left(0, h_{0}\right)$ :

$$
\begin{aligned}
\int_{-1-s}^{1-s} h^{2}\left|\partial_{x}\left(e^{\Phi_{2} / h} \psi_{s}\right)\right|^{2} \mathrm{~d} x & +\sum_{j= \pm} s_{j}^{2}\left(\pi^{2}-\rho^{2}\right)\left(D_{j}^{-2}-\pi^{2}-C h_{0}^{2 / 3}\right) \int_{I_{j}^{1}}\left|e^{\Phi_{2}^{j} / h} \psi_{s}\right|^{2} \mathrm{~d} x \\
& \leq C h_{0}^{2 / 3} \sum_{j= \pm} \int_{I_{j}^{2}}\left|e^{\Phi_{2}^{j} / h} \psi_{s}\right|^{2} \mathrm{~d} x
\end{aligned}
$$

$\Phi_{2}^{ \pm}$being non-positive on $I_{ \pm}^{2}$ we obtain the second inequality.

\subsection{Approximation of the first eigenfunctions by tensor products}

In this subsection we will work with the operator $\mathcal{L}_{\operatorname{Rec}(s)}(h)$ defined on the left side $u \leq 0$ by $\mathcal{L}_{s}^{\text {lef }}(h)$ and on the right side $u \geq 0$ by $\mathcal{L}_{s}^{\text {rig }}(h)$. Let us consider the $N_{0}$ first eigenvalues of $\mathcal{L}_{\operatorname{Rec}(s)}(h)$ (shortly denoted by $\left.\lambda_{s, n}(h)\right)$. In each corresponding eigenspace we choose a normalized eigenfunction $\hat{\psi}_{s, n}$ so that $\left\langle\hat{\psi}_{s, n}, \hat{\psi}_{s, p}\right\rangle=0$ if $n \neq p$. We introduce:

$$
\widehat{\mathcal{S}}_{s, N_{0}}(h)=\operatorname{span}\left(\hat{\psi}_{s, 1}, \ldots, \hat{\psi}_{s, N_{0}}\right) .
$$

Then, we follow the same lines as in [7, Sec. 4.3] and define the following quadratic form:

$$
\begin{aligned}
Q_{\operatorname{Rec}(s)}^{0}\left(\hat{\psi}_{s}\right)= & \int_{\mathrm{R}_{-}(s)}\left(\left|\partial_{t} \hat{\psi}_{s}\right|^{2}-\pi^{2}\left|\hat{\psi}_{s}\right|^{2}\right)\left(1+\frac{u}{s_{-}}\right) \mathrm{d} u \mathrm{~d} t+ \\
& \int_{\mathrm{R}_{+}(s)}\left(\left|\partial_{t} \hat{\psi}_{s}\right|^{2}-\pi^{2}\left|\hat{\psi}_{s}\right|^{2}\right)\left(1+\frac{u}{s_{+}}\right) \mathrm{d} u \mathrm{~d} t,
\end{aligned}
$$

where $\mathrm{R}_{-}(s)=\operatorname{Rec}(s) \cap\{u \leq 0\}$ and $\mathrm{R}_{+}(s)=\operatorname{Rec}(s) \cap\{u \geq 0\}$. We consider the projection:

$$
\Pi_{0} \hat{\psi}_{s}(u, t)=\left\langle\hat{\psi}_{s}(u, \cdot), \mathfrak{s}_{1}\right\rangle_{t} \mathfrak{s}_{1}(t) .
$$

We can now state a first approximation result: 
Proposition 3.7 Let $s_{0} \in(0,1)$, there exists $h_{0}>0$ and $C>0$ such that for all $s \in\left[-s_{0}, s_{0}\right]$, all $h \in\left(0, h_{0}\right)$ and all $\hat{\psi}_{s} \in \widehat{\mathcal{S}}_{s, N_{0}}(h)$ :

$$
0 \leq Q_{\operatorname{Rec}(s)}\left(\hat{\psi}_{s}\right) \leq C h^{2 / 3}\left\|\hat{\psi}_{s}\right\|^{2}
$$

and

$$
\left\|\left(\operatorname{Id}-\Pi_{0}\right) \hat{\psi}_{s}\right\|+\left\|\partial_{t}\left(\left(\operatorname{Id}-\Pi_{0}\right) \hat{\psi}_{s}\right)\right\| \leq C h^{1 / 3}\left\|\hat{\psi}_{s}\right\|
$$

Moreover we have, $\Pi_{0}: \widehat{\mathcal{S}}_{s, N_{0}} \rightarrow \Pi_{0}\left(\widehat{\mathcal{S}}_{s, N_{0}}\right)$ is an isomorphism.

Proof: We use the same reasoning as in [7, Sec 4.3] and [22, Sec 4.3].

\subsection{Reduction to the model operator}

The aim of this subsection is to prove Theorem 1.2 using the projections of the true eigenfunctions $\left(\Pi_{0} \widehat{\psi}_{s, n}\right)$ as test functions for the quadratic form of the model operator. We apply the technical background of [22, Sec. 4.4]. Let us consider $\widehat{\psi} \in \widehat{S}_{s, N_{0}}(h)$. We will need the two following lemmas to estimate the quadratic form of the model operator tested on $\left(\Pi_{0} \widehat{\psi}\right)$. The key in their proof is the use of Proposition 3.6.

Lemma 3.8 Let $s_{0} \in(0,1)$. There exist $h_{0}>0$ and $C>0$ such that for all $s \in\left[-s_{0}, s_{0}\right]$, all $h \in\left(0, h_{0}\right)$ and for all $\widehat{\psi} \in \widehat{\mathcal{S}}_{s, N_{0}}$ :

$$
\left|h^{2} \int_{\mathrm{R}_{ \pm}(s)} \partial_{u} \widehat{\psi}_{s} \partial_{t} \widehat{\psi}_{s} t\left(1+\frac{u}{s_{ \pm}}\right) \mathrm{d} u \mathrm{~d} t\right|^{2} \leq C h^{4 / 3}\left\|\widehat{\psi}_{s}\right\|^{2}
$$

Lemma 3.9 Let $s_{0} \in(0,1)$. There exist $h_{0}>0$ and $C>0$ such that for all $s \in\left[-s_{0}, s_{0}\right]$, all $h \in\left(0, h_{0}\right)$ and for all $\widehat{\psi} \in \widehat{\mathcal{S}}_{s, N_{0}}$ :

$$
\left.\left|\frac{h^{2}}{s_{ \pm}} \int_{\mathrm{R}_{ \pm}(s)}\right| \partial_{u} \widehat{\psi}_{s}\right|^{2}|u| \mathrm{d} u \mathrm{~d} t\left|\leq C h^{4 / 3}\left\|\widehat{\psi}_{s}\right\|^{2}, \quad\right| \frac{1}{s_{ \pm}^{2}} \int_{\mathrm{R}_{ \pm}(s)}\left|\widehat{\psi}_{s}\right|^{2}|u|^{2} \mathrm{~d} u \mathrm{~d} t \mid \leq C h^{4 / 3}\left\|\hat{\psi}_{s}\right\|^{2}
$$

We can now prove the

Proposition 3.10 Let $s_{0} \in(0,1)$. There exist $h_{0}>0$ and $C>0$ such that for all $s \in\left[-s_{0}, s_{0}\right]$, all $h \in\left(0, h_{0}\right)$ and for all $\widehat{\psi} \in \widehat{\mathcal{S}}_{s, N_{0}}$, we have:

$$
Q_{s, h}^{\bmod }\left(\hat{\psi}_{s}\right) \leq\left(\lambda_{s, N_{0}}(h)-\pi^{2}\right)\left\|\hat{\psi}_{s}\right\|^{2}+C h^{4 / 3}\left\|\hat{\psi}_{s}\right\|^{2}
$$

where

$$
Q_{s, h}^{\text {mod }}\left(\hat{\psi}_{s}\right):=\int_{\mathrm{R}_{-}(s)} h^{2}\left|\partial_{u} \hat{\psi}_{s}\right|^{2}+\frac{2 \pi^{2}}{1+s}|u|\left|\hat{\psi}_{s}\right|^{2} \mathrm{~d} u \mathrm{~d} t+\int_{\mathrm{R}_{+}(s)} h^{2}\left|\partial_{u} \hat{\psi}_{s}\right|^{2}+\frac{2 \pi^{2}}{1-s}|u|\left|\psi_{s}\right|^{2} \mathrm{~d} u \mathrm{~d} t .
$$


We remark that to study $Q_{s, h}^{\text {mod }}$ the variable $s$ and $t$ can be separated. In fact, in the $u$ variable, up to $h^{2}$ in front of the derivative term and a factor $2 \pi^{2}$ for the potential, $Q_{s, h}^{\text {mod }}$ is the quadratic form of the model operator in two dimension.

Proof: Let us take $\psi_{s} \in \mathcal{S}_{s, N_{0}}(h)$. As the $\left(\psi_{s, j}\right)_{j \in\left\{1, \ldots, N_{0}\right\}}$ are orthogonal, we have:

$$
Q_{s, h}\left(\psi_{s}\right) \leq \lambda_{s, N_{0}}(h)\left\|\psi_{s}\right\|^{2}
$$

By definition, for all $\psi \in \operatorname{Dom}\left(Q_{s, h}\right)$ we have:

$$
Q_{s, h}(\psi) \geq \int_{\operatorname{Tri}(s)} h^{2}\left|\partial_{x} \psi\right|^{2}+v_{s}(x)|\psi|^{2} \mathrm{~d} x \mathrm{~d} y .
$$

The last inequality combined with the convexity of the effective potential $v_{s}$ yields:

$$
\int_{\operatorname{Tri}(s)} h^{2}\left|\partial_{x} \psi_{s}\right|^{2}+2 \pi^{2}\left(\frac{\mathbb{1}_{\mathbb{R}_{-}}(x)}{1+s}+\frac{\mathbb{1}_{\mathbb{R}_{-}}(x)}{1-s}\right)|x|\left|\psi_{s}\right|^{2} \mathrm{~d} x \mathrm{~d} y \leq\left(\lambda_{s, N_{0}}(h)-\pi^{2}\right)\left\|\psi_{s}\right\|^{2} .
$$

Then, we perform the change of variables (3.4) to get:

$$
\begin{aligned}
Q_{s, h}^{\bmod }\left(\hat{\psi}_{s}\right) \leq & \left(\lambda_{s, N_{0}}(h)-\pi^{2}\right)\left\|\hat{\psi}_{s}\right\|^{2}+\frac{h^{2}}{s_{-}} \int_{\mathrm{R}_{-}(s)}\left|\hat{\psi}_{s}\right|^{2}|u| \mathrm{d} u \mathrm{~d} t+\frac{h^{2}}{\left|s_{+}\right|} \int_{\mathrm{R}_{+}(s)}\left|\hat{\psi}_{s}\right|^{2}|u| \mathrm{d} u \mathrm{~d} t+ \\
& \frac{2 \pi^{2}}{s_{-}^{2}} \int_{\mathrm{R}_{-}(s)}\left|\hat{\psi}_{s}\right|^{2}|u|^{2} \mathrm{~d} u \mathrm{~d} t+\frac{2 \pi^{2}}{s_{+}^{2}} \int_{\mathrm{R}_{+}(s)}\left|\hat{\psi}_{s}\right|^{2}|u|^{2} \mathrm{~d} u \mathrm{~d} t+ \\
& 2 h^{2} \int_{\mathrm{R}_{-}(s)} t \partial_{u} \hat{\psi}_{s} \partial_{t} \hat{\psi}_{s}\left(1+\frac{u}{s_{-}}\right) \mathrm{d} u \mathrm{~d} t-2 h^{2} \int_{\mathrm{R}_{+}(s)} t \partial_{u} \hat{\psi}_{s} \partial_{t} \hat{\psi}_{s}\left(1+\frac{u}{s_{+}}\right) \mathrm{d} u \mathrm{~d} t
\end{aligned}
$$

To obtain Proposition 3.10 we apply Lemmas 3.8 and 3.9 taking into account (3.11).

Proof of Theorem 1.2 We apply Proposition 3.7 to the result of Proposition 3.10 and we obtain:

$$
Q_{s, h}^{\bmod }\left(\hat{\psi}_{s}\right) \leq\left(\lambda_{s, N_{0}}(h)-\pi^{2}\right)\left\|\Pi_{0} \hat{\psi}_{s}\right\|^{2}+C h^{4 / 3}\left\|\Pi_{0} \hat{\psi}_{s}\right\|^{2} .
$$

Then, equation (3.11) and Lemma 3.7 yield:

$$
Q_{s, h}^{\bmod }\left(\hat{\psi}_{s}\right) \leq\left(\lambda_{s, N_{0}}(h)-\pi^{2}\right)\left\|\Pi_{0} \hat{\psi}_{s}\right\|_{L^{2}(\operatorname{Rec}(s))}^{2}+C h^{4 / 3}\left\|\Pi_{0} \hat{\psi}_{s}\right\|_{L^{2}(\operatorname{Rec}(s))}^{2}
$$

where

$$
\left\|\hat{\psi}_{s}\right\|_{L^{2}(\operatorname{Rec}(s))}^{2}=\left\|\hat{\psi}_{s}\right\|_{L^{2}\left(\mathrm{R}_{-}(s), \mathrm{d} u \mathrm{~d} t\right)}^{2}+\left\|\hat{\psi}_{s}\right\|_{L^{2}\left(\mathrm{R}_{+}(s), \mathrm{d} u \mathrm{~d} t\right)}^{2} .
$$

Moreover, we have

$$
Q_{s, h}^{\bmod }\left(\hat{\psi}_{s}\right)=Q_{s, h}^{\bmod }\left(\Pi_{0} \hat{\psi}_{s}\right)+Q_{s, h}^{\bmod }\left(\left(\operatorname{Id}-\Pi_{0}\right) \hat{\psi}_{s}\right)+2 b_{s, h}^{\bmod }\left(\Pi_{0} \hat{\psi}_{s},\left(\operatorname{Id}-\Pi_{0}\right) \hat{\psi}_{s}\right),
$$

where $b_{s, h}^{\text {mod }}$ is the bilinear form associated with $Q_{s, h}^{\text {mod }}$. As the variable $u$ and $t$ can be separated we remark that

$$
b_{s, h}^{\bmod }\left(\Pi_{0} \hat{\psi}_{s},\left(\operatorname{Id}-\Pi_{0}\right) \hat{\psi}_{s}\right)=0 .
$$

Finally we have

$$
Q_{s, h}^{\bmod }\left(\Pi_{0} \hat{\psi}_{s}\right) \leq\left(\lambda_{s, N_{0}}(h)-\pi^{2}\right)\left\|\Pi_{0} \hat{\psi}_{s}\right\|_{L^{2}(\operatorname{Rec}(s))}^{2}+C h^{4 / 3}\left\|\Pi_{0} \hat{\psi}_{s}\right\|_{L^{2}(\operatorname{Rec}(s))}^{2} .
$$


Now the conclusion is standard. We denote by $\pi_{0} \hat{\psi}_{s}:=\left\langle\hat{\psi}_{s}(u, \cdot), \mathfrak{s}_{1}\right\rangle_{t}$. Let us consider the smooth cut-off function $\chi$ being 1 for $|u| \leq \frac{1}{4}$ and 0 for $|u| \geq \frac{1}{2}$. We define $\chi_{s}(u):=\chi\left(\frac{u}{1-s}\right)$ it holds:

$$
q_{s, h}^{\bmod }\left(\chi_{s} \pi_{0} \hat{\psi}_{s}\right) \leq\left(\lambda_{s, N_{0}}(h)-\pi^{2}\right)\left\|\pi_{0} \hat{\psi}_{s}\right\|^{2}+C h^{4 / 3}\left\|\pi_{0} \hat{\psi}_{s}\right\|^{2}
$$

where

$$
q_{s, h}^{\bmod }(\varphi):=\int_{-1-s}^{1-s} h^{2}\left|\partial_{u} \varphi\right|^{2}+2 \pi^{2}\left(\frac{\mathbb{1}_{\mathbb{R}_{-}}(u)}{1+s}+\frac{\mathbb{1}_{\mathbb{R}_{-}}(u)}{1-s}\right)|u||\varphi|^{2} \mathrm{~d} u .
$$

Then, we consider $\widehat{s}_{s, N_{0}}(h):=\operatorname{span}\left(\pi_{0} \hat{\psi}_{s, 1}, \ldots, \pi_{0} \hat{\psi}_{s, N_{0}}\right)$ and we apply the min-max principle to the $N_{0}$ dimensional space $\chi_{s} \widehat{s}_{s, N_{0}}(h)$ wich yields

$$
\pi^{2}+\left(2 \pi^{2}\right)^{2 / 3} \kappa_{N_{0}}(s) h^{2 / 3} \leq \lambda_{s, N_{0}}(h) .
$$

Jointly with Proposition 3.1, this finishes the proof of Theorem 1.2.

\section{An application: the mountain $\Omega(h)$}

The aim of this section is to prove Theorem 1.4. To do so, we follow the philosophy of [13]. After the proof of some Agmon localization estimates in Subsection 4.1 we prove Theorem 1.4 in Subsection 4.2. To avoid the dependence on $h$ of the domain $\Omega(h)$ we perform the scaling:

$$
x=x_{1} ; \quad y=\frac{1}{h} x_{2} .
$$

The domain $\Omega(h)$ is now $\Omega:=\Omega(1)$ and we respectively denote by $\mathcal{L}(h), \mathcal{L}^{\text {lef }}(h), \mathcal{L}^{\text {rig }}(h)$ and $\mathfrak{L}(h)$ the operators $-\Delta_{\Omega(h)}^{\mathrm{Dir}},-\Delta_{\Omega^{\mathrm{lef}}(h)}^{\mathrm{Dir}},-\Delta_{\Omega^{\mathrm{rig}}(h)}^{\mathrm{Dir}}$ and $\mathfrak{D}(h)$ in this new variables, up to a multiplication by $h^{2} \cdot \mathcal{L}(h), \mathcal{L}^{\text {lef }}(h)$ and $\mathcal{L}^{\text {rig }}(h)$ are the Dirichlet realization of $-h^{2} \partial_{x}^{2}+\partial_{y}^{2}$ on each associated geometric domain (denoted $\Omega, \Omega^{\text {lef }}$ and $\Omega^{\text {rig }}$ ). We denote by $\left(\lambda_{n}(h)\right)_{n \geq 1}$ the eigenvalues of $\mathcal{L}(h)$, whereas the eigenvalues of the two operators $\mathcal{L}^{\text {lef }}(h)$ and $\mathcal{L}^{\text {rig }}(h)$ are respectively denoted by $\left(\lambda_{n}^{\text {lef }}(h)\right)_{n \geq 1}$ and $\left(\lambda_{n}^{\text {rig }}(h)\right)_{n \geq 1}$. In terms of physical variables, we obtain:

$$
\mu_{n}(h)=h^{-2} \lambda_{n}(h) ; \quad \mu_{n}^{\text {lef }}(h)=h^{-2} \lambda_{n}^{\text {lef }}(h) ; \quad \mu_{n}^{\text {rig }}(h)=h^{-2} \lambda_{n}^{\text {rig }}(h) .
$$

We also denote by $\left(\omega_{n}(h)\right)_{n \geq 1}$ the eigenvalues of $\mathfrak{L}(h)$ and they satisfy $\nu_{n}(h)=h^{-2} \omega_{n}(h)$.

As in Subsection 1.4, we construct for $\mathcal{L}(h)$ an effective potential in the spirit of the BornOppenheimer approximation. We obtain the operator:

$$
-h^{2} \partial_{x}^{2}+v(x)
$$

where the effective potential $v$ is:

$$
\begin{aligned}
\pi^{-2} v(x)= & \left(\frac{1-s^{\text {lef }}}{\tau(x+1)}\right)^{2} \mathbb{1}_{\left(-1,-s^{\text {lef }}\right)}(x)+\left(\frac{s^{\text {lef }}}{-(\tau-\varsigma) x+\varsigma s^{\text {lef }}}\right)^{2} \mathbb{1}_{\left(-s^{\text {lef }}, 0\right)}(x) \\
& +\left(\frac{s^{\text {rig }}}{(1-\varsigma) x+\varsigma s^{\text {rig }}}\right)^{2} \mathbb{1}_{\left(0, s^{\text {rig }}\right)}(x)+\left(\frac{1-s^{\text {rig }}}{x-1}\right)^{2} \mathbb{1}_{\left(s^{\text {rig }, 1)}\right.}(x) .
\end{aligned}
$$

The shape of the potential helps us to get the Agmon estimates of Subsection 4.1. 


\subsection{Agmon estimates}

To go further, we need Agmon localization estimates on $\Omega$ for the true eigenfunctions of $\mathcal{L}(h), \mathcal{L}^{\text {lef }}(h)$ and $\mathcal{L}^{\text {rig }}(h)$.

Agmon estimates for $\mathcal{L}^{\text {lef }}(h)$ and $\mathcal{L}^{\text {rig }}(h) \quad$ The shape of the effective potentials associated with $\mathcal{L}^{\text {lef }}(h)$ and $\mathcal{L}^{\text {rig }}(h)$ is the same as the one of $v_{s}$ in (1.1). Consequently, the Agmon estimates near $x=-s^{\text {lef }}$ and $x=s^{\text {rig }}$ are obtained exactly as in the proof of Proposition 3.6. It yields the

Proposition 4.1 Let $\Gamma_{0}>0$. There exist $h_{0}>0, C_{0}>0$ and $C>0$ such that for all $h \in\left(0, h_{0}\right)$ and all eigenpair $\left(\lambda^{\text {lef }}, \psi\right)$ of $\mathcal{L}^{\text {lef }}(h)$ satisfying $\left|\lambda^{\text {lef }}-\pi^{2}\right| \leq \Gamma_{0} h^{2 / 3}$, we have:

$$
\int_{\Omega^{\text {lef }} \cap\left\{\left(-s^{\text {lef }} / 2,0\right) \times \mathbb{R}\right\}}|\psi|^{2}+\left|h^{2 / 3} \partial_{x} \psi\right|^{2} \mathrm{~d} x \mathrm{~d} y \leq C_{0} e^{-C / h}\|\psi\|^{2} .
$$

The same proposition holds for $\mathcal{L}^{\text {rig }}(h)$.

Remark 4.2 The domain $\Omega^{\text {lef }}$ can be considered as a truncated triangle. Let us denote by Trilef this triangle (see Figure 4). We define $\mathcal{L}_{\text {Trilef }}(h)$ as the Dirichlet realization on Tri ${ }^{\text {lef }}$ of $-h^{2} \partial_{x}^{2}-\partial_{y}^{2}$.

Thanks to Proposition 4.1 one can prove that if $(\lambda, \psi)$ is an eigenpair of $\mathcal{L}^{\text {lef }}(h)$ there exists $C>0$ such that:

$$
\left\|\left(\mathcal{L}_{\text {Trief }}(h)-\lambda\right) \psi\right\|=\mathcal{O}\left(e^{-C / h}\right) .
$$

Reciprocally, let $(\lambda, \psi)$ be an eigenpair of $\mathcal{L}_{\text {Trief }}(h)$. Using a rescaled version of Proposition 3.6, for a well chosen cut-off function $\chi$, we have:

$$
\left\|\left(\mathcal{L}^{\text {lef }}(h)-\lambda\right)(\chi \psi)\right\|=\mathcal{O}\left(e^{-C / h}\right) .
$$

Thanks to the spectral theorem, we know that for $n \in \mathbb{N}^{*}$ the $n$-th eigenpair of $\mathcal{L}_{\text {Trief }}(h)$ and the $n$-th eigenpair of $\mathcal{L}^{\text {lef }}(h)$ are the same up to a remainder of order $\mathcal{O}\left(e^{-C / h}\right)$. Besides, thanks to a rescaled version of Theorem 1.2 we have an asymptotic expansion of the eigenpairs of $\mathcal{L}_{\text {Trief }}(h)$. Consequently, the same asymptotic expansion holds for the eigenpairs of $\mathcal{L}^{\text {lef }}(h)$.

In fact, in the semiclassical limit $h \rightarrow 0$, it is the local structure of the peak that leads the asymptotic expansion of the eigenpairs.

The same reasoning holds for the problem on the right.

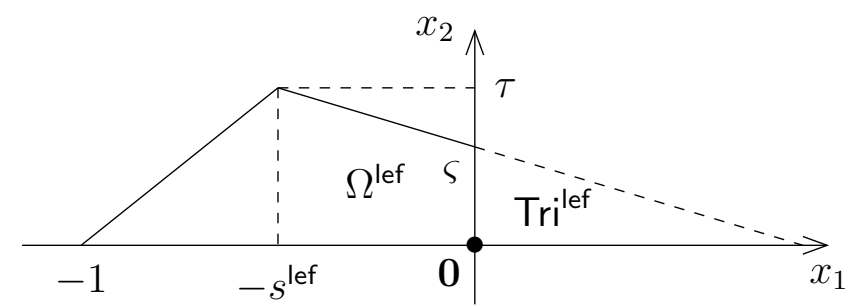

Figure 4: The domain $\Omega^{\text {lef }}$ and the triangle Tri ${ }^{\text {lef }}$. 
Agmon estimates for $\mathcal{L}(h)$ between the two peaks We can obtain Agmon estimates for $\mathcal{L}(h)$ near each peak $\left(x=-s^{\text {lef }}\right.$ and $\left.x=-s^{\text {rig }}\right)$ following the ideas of the proof of Proposition 3.6. It is the shape of the effective potential $v$ in (4.1) that leads to the result. Finally we obtain the

Proposition 4.3 Let $\Gamma_{0}>0$. There exist $h_{0}>0, C_{0}>0$ and $C>0$ such that for all $h \in\left(0, h_{0}\right)$ and all eigenpair $(\lambda, \psi)$ of $\mathcal{L}(h)$ satisfying $\left|\lambda-\pi^{2}\right| \leq \Gamma_{0} h^{2 / 3}$, we have:

$$
\begin{gathered}
\int_{\Omega \cap\left\{\left(-1, s^{\text {rig }} / 2\right) \times \mathbb{R}\right\}}|\psi|^{2}+\left|h^{2 / 3} \partial_{x} \psi\right|^{2} \mathrm{~d} x \mathrm{~d} y \leq C_{0} e^{-C / h}\|\psi\|^{2}, \text { when } \tau<1, \\
\int_{\Omega \cap\left\{\left(-s^{\text {lef }} / 2, s^{\text {rig }} / 2\right) \times \mathbb{R}\right\}}|\psi|^{2}+\left|h^{2 / 3} \partial_{x} \psi\right|^{2} \mathrm{~d} x \mathrm{~d} y \leq C_{0} e^{-C / h}\|\psi\|^{2}, \text { when } \tau=1 .
\end{gathered}
$$

We recall that when $\tau<1$, the right peak is higher than the one on the left. Proposition 4.3 says that the eigenfunctions are mainly localized near the highest peak. When $\tau=1$, they have the same height and Proposition 4.3 means that the eigenfunctions are mainly localized near the altitudes from $C_{1}$ and $C_{2}$.

\subsection{Spectrum of $\mathcal{L}(h)$}

The aim of this subsection is to prove Theorem 1.4. To do so we use the eigenpairs of $\mathfrak{L}(h)$ as quasimodes for the operator $\mathcal{L}(h)$. Then, we do the same thing permuting the roles of $\mathfrak{L}(h)$ and $\mathcal{L}(h)$.

Proof of Theorem 1.4 Let $N_{0} \in \mathbb{N}^{*}$, if $\left(\omega_{j}(h), \psi_{h, j}\right)$ is an eigenpair of $\mathfrak{L}(h)$. This eigenpair is an eigenpair of $\mathcal{L}^{\text {lef }}(h)$ or $\mathcal{L}^{\text {rig }}(h)$. The proof being the same replacing $\mathcal{L}^{\text {rig }}(h)$ by $\mathcal{L}^{\text {lef }}(h)$, we assume that $\left(\omega_{j}(h), \psi_{h, j}\right)$ is an eigenpair of $\mathcal{L}^{\text {rig }}(h)$. Now, we consider a smooth cut-off function $\chi^{\text {rig }}$, such that:

$$
\chi^{\text {rig }}(x)=1 \text { if } x \geq s^{\text {rig }} / 2, \chi^{\text {rig }}(x)=0 \text { if } x \leq s^{\text {rig }} / 4 .
$$

Thanks to Proposition 4.1, it is not hard to prove that $\left\|\chi^{\text {rig }} \psi_{h, j}\right\|=\left\|\psi_{h, j}\right\|\left(1+\mathcal{O}\left(e^{-C / h}\right)\right) \neq 0$. We remark that:

$$
\left(\mathcal{L}(h)-\omega_{j}(h)\right) \chi^{\mathrm{rig}} \psi_{h, j}=\left(\mathcal{L}^{\mathrm{rig}}(h)-\omega_{j}(h)\right) \chi^{\mathrm{rig}} \psi_{h, j} .
$$

Consequently, thanks to the Agmon estimates of Proposition 4.1, we obtain the existence of $C_{0}, C>0$, such that:

$$
\left\|\left(\mathcal{L}(h)-\omega_{j}(h)\right) \chi^{\mathrm{rig}} \psi_{h, j}\right\| \leq C_{0} e^{-C / h}\left\|\chi^{\mathrm{rig}} \psi_{h, j}\right\| .
$$
that:

Now, let $\left(\lambda_{j}(h), \Psi_{h, j}\right)$ be an eigenpair of $\mathcal{L}(h)$. We consider a smooth cut-off function $\chi^{\text {lef }}$, such

$$
\chi^{\text {lef }}(x)=1 \text { if } x \leq-s^{\text {lef }} / 4, \chi^{\text {rig }}(x)=0 \text { if } x \geq-s^{\text {lef }} / 4 .
$$

Thanks to Proposition 4.3, it is not hard to prove that

$$
\left\|\left(\chi^{\text {lef }} \Psi_{h, j}, \chi^{\text {rig }} \Psi_{h, j}\right)\right\|_{L^{2}\left(\Omega^{\text {lef }}\right) \oplus L^{2}\left(\Omega^{\text {rig }}\right)}=\left\|\Psi_{h, j}\right\|\left(1+\mathcal{O}\left(e^{-C / h}\right)\right) \neq 0 .
$$

We remark that:

$$
\begin{aligned}
\left\|\left(\mathfrak{L}(h)-\lambda_{j}(h)\right)\left(\chi^{\text {lef }} \Psi_{h, j}, \chi^{\text {rig }} \Psi_{h, j}\right)\right\|_{L^{2}\left(\Omega^{\text {lef }}\right) \oplus L^{2}\left(\Omega^{\text {rig }}\right)}^{2}= & \left\|\left(\mathcal{L}^{\text {lef }}(h)-\lambda_{j}(h)\right) \chi^{\text {lef }} \Psi_{h, j}\right\|_{L^{2}\left(\Omega^{\text {lef }}\right)}^{2} \\
& +\left\|\left(\mathcal{L}^{\text {rig }}(h)-\lambda_{j}(h)\right) \chi^{\text {rig }} \Psi_{h, j}\right\|_{L^{2}\left(\Omega^{\text {rig }}\right)}^{2} \\
= & \left\|\left(\mathcal{L}(h)-\lambda_{j}(h)\right)\left(\chi^{\text {lef }} \Psi_{h, j}+\chi^{\text {rig }} \Psi_{h, j}\right)\right\|^{2}
\end{aligned}
$$


Thanks to the Agmon estimates of Proposition 4.3 we obtain the existence of $C_{0}, C>0$, such that:

$$
\left\|\left(\mathfrak{L}(h)-\lambda_{j}(h)\right)\left(\chi^{\text {lef }} \Psi_{h, j}, \chi^{\text {rig }} \Psi_{h, j}\right)\right\|_{L^{2}\left(\Omega^{\text {lef }}\right) \oplus L^{2}\left(\Omega^{\text {rig }}\right)} \leq C_{0} e^{-C / h}\left\|\left(\chi^{\text {lef }} \Psi_{h, j}, \chi^{\text {rig }} \Psi_{h, j}\right)\right\|_{L^{2}\left(\Omega^{\text {lef }}\right) \oplus L^{2}\left(\Omega^{\text {rig }}\right)} .
$$

Combining (4.2) and (4.3), the spectral theorem gives Theorem 1.4.

In Appendix B we explicit Theorem 1.4 in different cases and illustrate each case with numerical simulations.

\section{A About the triangle: Shape of the eigenfunctions in the semiclas- sical limit}

Let us now illustrate some theoretical properties of the eigenfunctions of $\mathcal{L}_{s}(h)$ with numerical simulations. These computations are performed in the domain Tri $(s)$ with the finite element library Melina++ [18]. The mesh is constituted of triangles with 4 subdivisions on $\operatorname{Tri}^{-}(s)$ and $\mathrm{Tri}^{+}(s)$ with 6 as interpolation degree. Figure 5 pictures the dominant term in the construction (3.10): It is almost a tensor product of the eigenfunction of the model operator and the sinus (respectively along the $X$-axis and the $Y$-axis). The eigenfunctions are localized near the altitude of the triangle. This matches with the Agmon estimates of Proposition 3.6.

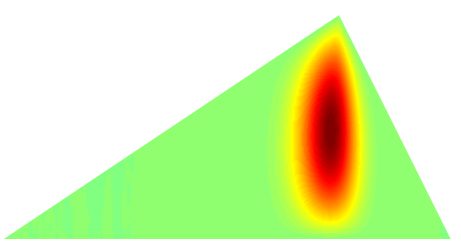

$$
\lambda_{1}(s, h)=14.119615
$$

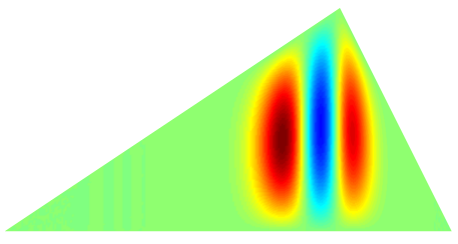

$$
\lambda_{3}(s, h)=18.59000
$$

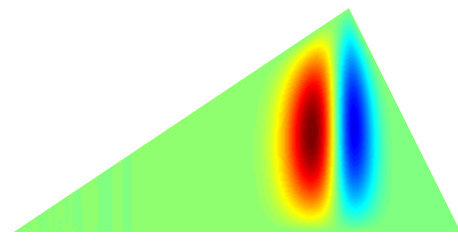

$\lambda_{2}(s, h)=16.461797$

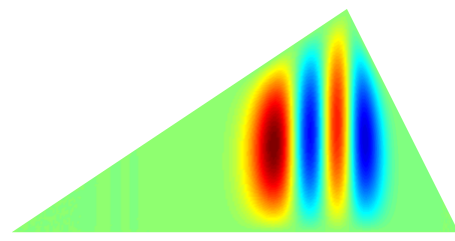

$\lambda_{4}(s, h)=20.63603$

Figure 5: This figure represents the four first eigenfunctions of $\mathcal{L}_{s}(h)$ and their corresponding eigenvalue for $s=0.5$ and $h=0.1$. 


\section{B About the mountain with two peaks: Behavior of the eigenval- ues}

In this appendix we discuss the meaning of Theorem 1.4 in three particular cases. The computations exposed in this appendix are performed with the finite element library Melina++ [18]. The mesh is constituted of triangles with 4 subdivisions on seven initial triangles and with 6 as interpolation degree.

Depending on the structure of the peaks, in the semiclassical limit $h \rightarrow 0$, the eigenvalues $\left(\omega_{j}(h)\right)_{j \geq 1}$ of $\mathcal{L}(h)$ can be investigate thanks to Theorem 1.4.

In the following sections $N_{0}$ is a fixed positive integer.

\section{B.1 Two peaks with different height}

In this case, thanks to Theorem 1.2, we know that for all $j \in\left\{0, \ldots, N_{0}\right\}$, we have:

$$
\lambda_{j}^{\mathrm{rig}}(h)-\pi^{2} \underset{h \rightarrow 0}{=}\left(\frac{1}{\tau}-1\right) \pi^{2}+\mathcal{O}\left(h^{2 / 3}\right), \quad \lambda_{j}^{\text {lef }}(h)-\pi^{2} \underset{h \rightarrow 0}{=} \mathcal{O}\left(h^{2 / 3}\right) .
$$

So, for $h$ small enough, we have:

$$
\omega_{j}(h)=\lambda_{j}^{\operatorname{lef}}(h)+\mathcal{O}\left(e^{-C / h}\right) .
$$

When there is a higher peak, Theorem 1.4 means that in the semiclassical limit, the problem with two peaks is exponentially close to the problem with only the higher peak.

That does not mean that the asymptotics of the lowest peak does not play a role for $h$ large enough. In Figure 6 we represented the eight first eigenvalues of $\mathcal{L}(h)$ as functions of $h$, with $s^{\text {lef }}=s^{\text {rig }}=0.5$, $\tau=0.85$ and $\varsigma=0.5$. We observe that the asymptotics for the lower peak plays a role: If we start from $h=0.1$ with the third eigenvalue (the yellow one), it turns out that there is a value of $h$ for which it meets the forth one (and so on) to converge to $\frac{\pi^{2}}{\tau^{2}}$. In Figure 7, we zoomed near one of these points to illustrate numerically that it is a crossing. In Figure 8 we pictured the eigenfunctions associated with the eigenvalues of Figure 7 before and after the crossing. It turns out that the quasimodes built for each peak are good approximation of each mode: Before the crossing $(h=0: 01915)$, the seventh eigenfunction (respectively the eighth eigenfunction) is associated with the the first mode of the left peak alone (respectively the seventh mode of the right peak alone). After the crossing the role are inverted.

\section{B.2 Two non-isometric peaks with same heights}

In this case, thanks to Theorem 1.2, we know that for all $j \in\left\{0, \ldots, N_{0}\right\}$, we have:

$$
\lambda_{j}^{\mathrm{rig}}(h)-\pi^{2} \underset{h \rightarrow 0}{=} \kappa_{j}^{\text {lef }}\left(\varsigma, s^{\mathrm{lef}}\right) h^{2 / 3}+\mathcal{O}\left(h^{4 / 3}\right), \quad \lambda_{j}^{\mathrm{lef}}(h)-\pi^{2} \underset{h \rightarrow 0}{=} \kappa_{j}^{\text {rig }}\left(\varsigma, s^{\mathrm{rig}}\right) h^{2 / 3}+\mathcal{O}\left(h^{4 / 3}\right),
$$

where $\kappa_{j}^{\text {lef }}$ and $\kappa_{j}^{\text {rig }}$ are coefficients depending on the slopes of each peak. Assume that for $j, p \in \mathbb{N}^{*}$ we have $\kappa_{j}^{\text {lef }}\left(\varsigma, s^{\text {lef }}\right) \neq \kappa_{p}^{\text {rig }}\left(\varsigma, s^{\text {rig }}\right)$. Let us define $\mathrm{K}=\left\{\kappa_{j}^{\text {lef }}\left(\varsigma, s^{\text {lef }}\right)\right\}_{j \geq 1} \cup\left\{\kappa_{j}^{\text {rig }}\left(\varsigma, s^{\text {rig }}\right)\right\}_{j \geq 1}$ and $k_{j}$ the $j$-th element of $K$, counted in non-decreasing order. For $h$ small enough, $\omega_{j}(h)$ is the eigenvalue of $\mathcal{L}^{\text {lef }}(h)$ or $\mathcal{L}^{\text {lef }}(h)$ associated with the asymptotic expansion that begins as:

$$
\pi^{2}+k_{j} h^{2 / 3}+\mathcal{O}\left(h^{4 / 3}\right) .
$$




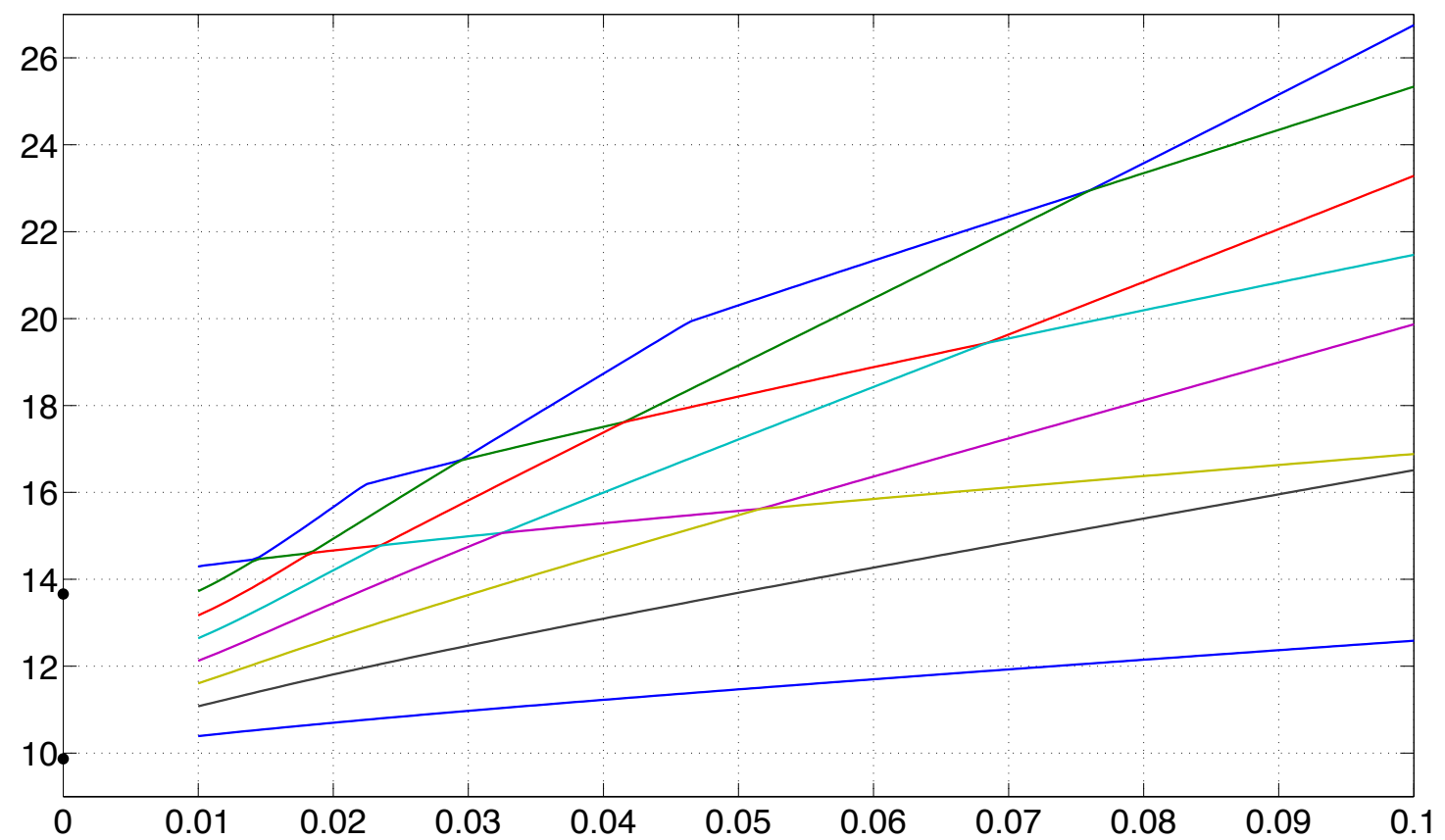

Figure 6: Eight first eigenvalues of $\mathcal{L}(h)$ as functions of $h$ with $s^{\text {lef }}=s^{\text {rig }}=0.5, \tau=0.85$ and $\varsigma=0.5$. The black dots are $\pi^{2}$ and $\frac{\pi^{2}}{\tau^{2}}$.

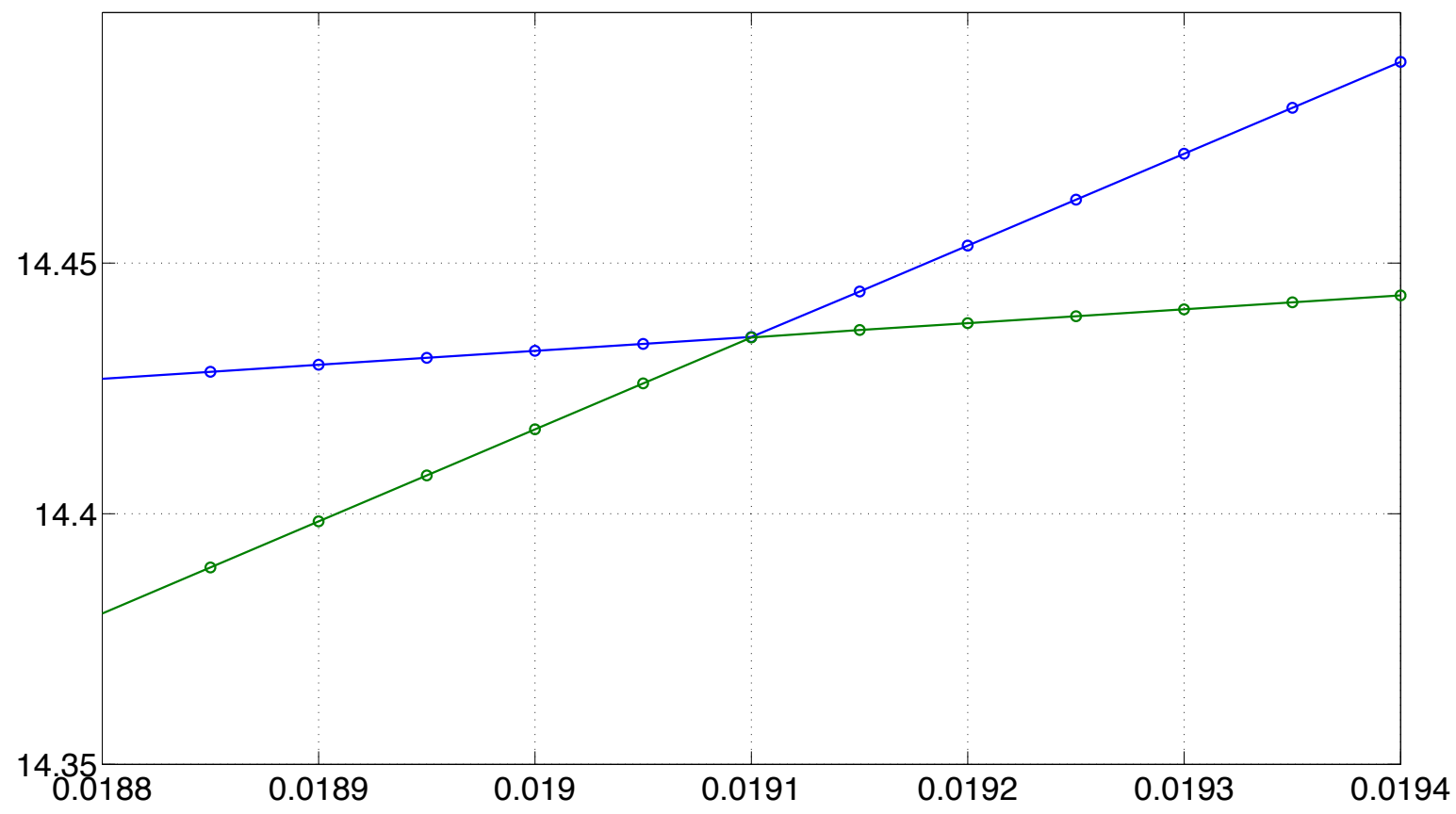

Figure 7: Zoom of Figure 6. 


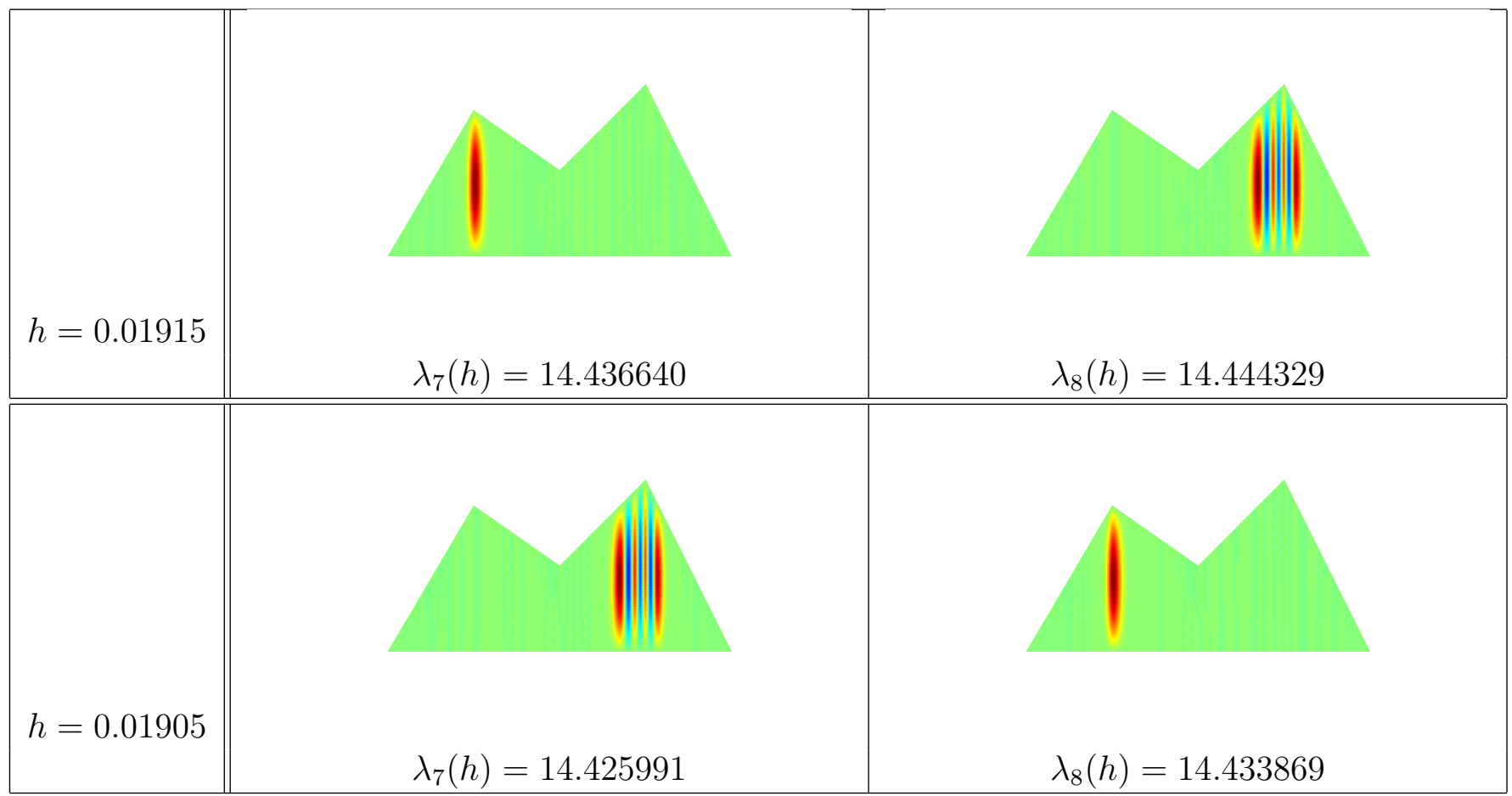

Figure 8: This figure represents the seventh and eighth eigenfunctions of $\mathcal{L}(h)$ and their corresponding eigenvalue for different values of $h$ with $s^{\text {lef }}=s^{\text {rig }}=0.5, \tau=0.85$ and $\varsigma=0.5$.

So, when there is two non-isometric peaks with same height satisfying for $j, p \in \mathbb{N}^{*}, \kappa_{j}^{\text {lef }}\left(\varsigma, s^{\text {lef }}\right) \neq$ $\kappa_{p}^{\text {rig }}\left(\varsigma, s^{\text {rig }}\right)$, the eigenvalues are alternatively exponentially close to the problem on the right and on the left, depending on the comparison of the coefficients of $\mathrm{K}$. We illustrated this property in Figure 9, with $s^{\text {lef }}=0.5, s^{\text {rig }}=0.25, \tau=1$ and $\varsigma=0.25$.

The situation $\kappa_{j}^{\text {lef }}\left(\varsigma, s^{\text {lef }}\right)=\kappa_{p}^{\text {rig }}\left(\varsigma, s^{\text {rig }}\right)$ is also interesting. We do not deal with it in the present paper but first, to understand it, one could find for which $s^{\text {rig }}, s^{\text {rig }}$ and $\varsigma$ this equality could hold.

\section{B.3 Two isometric peaks with same height}

In this case, the coefficients $\kappa_{j}^{\text {lef }}$ and $\kappa_{j}^{\text {rig }}$ satisfy $\kappa_{j}^{\text {lef }}\left(\varsigma, s^{\text {lef }}\right)=\kappa_{j}^{\text {rig }}\left(\varsigma, s^{\text {rig }}\right)$. Thanks to Theorem 1.2 we know that for all $j \in\left\{0, \ldots, N_{0}\right\}$ :

$$
\lambda_{j}^{\text {rig }}(h)-\lambda_{j}^{\text {lef }}(h) \underset{h \rightarrow 0}{=} \mathcal{O}\left(h^{\infty}\right) .
$$

Consequently, the spectrum of $\mathfrak{L}(h)$ can be seen as pairs of eigenvalues exponentially close. Theorem 1.4 yields the structure of the eigenvalues of $\mathcal{L}(h)$ and we notice a tunneling phenomenon. When $s^{\text {lef }}=s^{\text {rig }}$, the peaks are symmetric. In this case the result of Theorem 1.4 is reminiscent of the problem with symmetric electric potentials studied by Helffer [12] and Helffer and Sjöstrand [13] where the exponential gap between two exponentially close eigenvalues is given. Nevertheless, to do so, they need WKB constructions for non-degenerated potentials. Here, the effective potential associated with the problem is not smooth at $x=-s^{\text {rig }}$ and $x=s^{\text {rig. }}$. To obtain the exponential gap, a first hint would be the obtention of WKB construction in such a non-smooth well. 


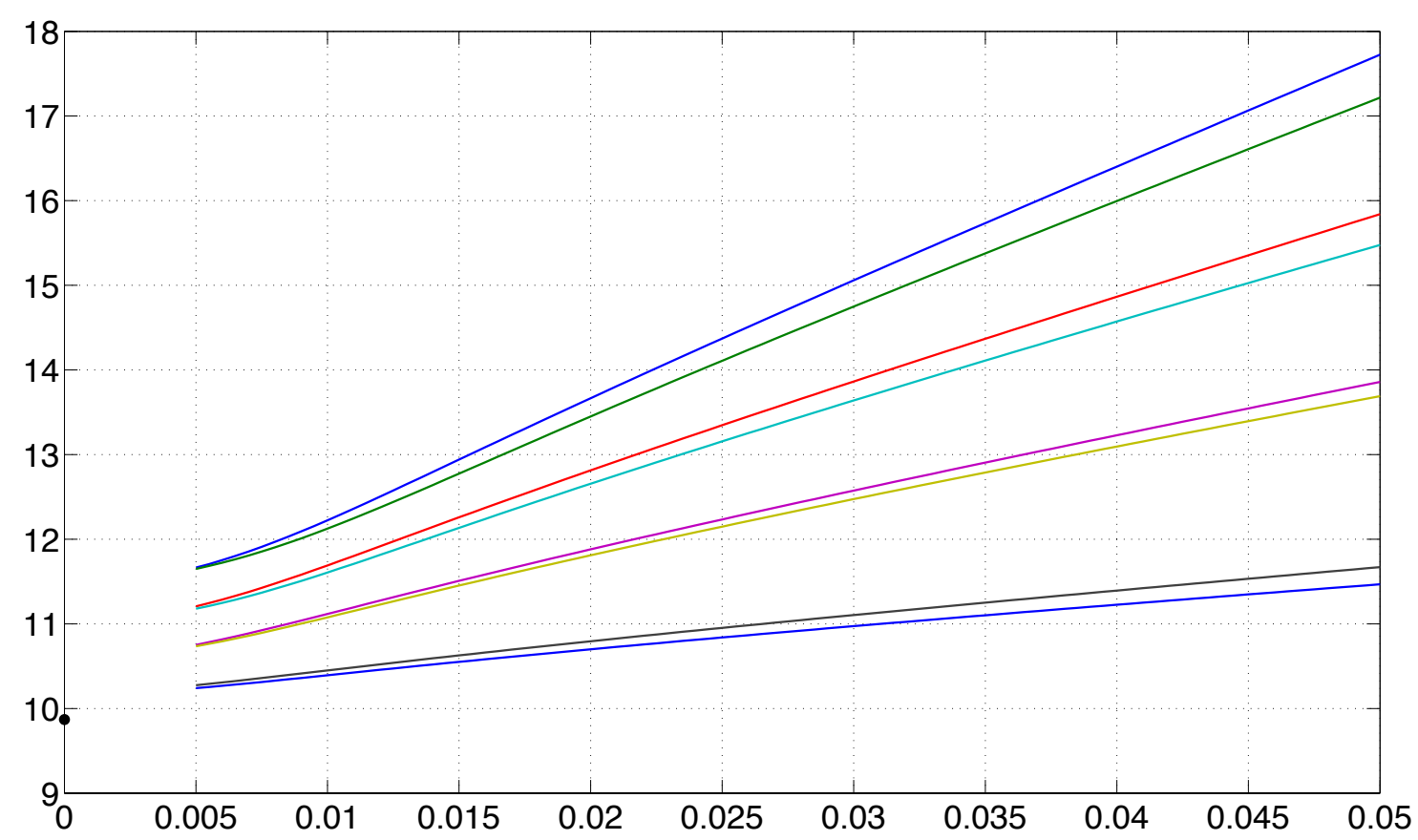

Figure 9: Eight first eigenvalues of $\mathcal{L}(h)$ as functions of $h$ with $s^{\text {lef }}=0.5, s^{\text {rig }}=0.25, \tau=1$ and $\varsigma=0.25$. The black dot is $\pi^{2}$.

Acknowledgment I would like to thank Luc Hillairet who gives the impulse to write this paper as well as Monique Dauge and Nicolas Raymond for all their advices on this subject. My thanks also go to Yvon Lafranche who built a mesh constructor adapted to this problem for the finite element library Melina++ [18].

\section{References}

[1] M. Abramowitz and I. A. Stegun. Handbook of mathematical functions with formulas, graphs, and mathematical tables, volume 55 of National Bureau of Standards Applied Mathematics Series. For sale by the Superintendent of Documents, U.S. Government Printing Office, Washington, D.C., 1964.

[2] S. Agmon. Lectures on exponential decay of solutions of second-order elliptic equations: bounds on eigenfunctions of $N$-body Schrödinger operators, volume 29 of Mathematical Notes. Princeton University Press, Princeton, NJ, 1982.

[3] S. Agmon. Bounds on exponential decay of eigenfunctions of Schrödinger operators. In Schrödinger operators (Como, 1984), volume 1159 of Lecture Notes in Math., pages 1-38. Springer, Berlin, 1985.

[4] D. Borisov and P. Freitas. Singular asymptotic expansions for Dirichlet eigenvalues and eigenfunctions of the Laplacian on thin planar domains. Ann. Inst. H. Poincaré Anal. Non Linéaire, 26(2):547-560, 2009. 
[5] J. Combes, P. Duclos, and R. Seiler. The born-oppenheimer approximation. In G. Velo and A. Wightman, editors, Rigorous Atomic and Molecular Physics, volume 74 of NATO Advanced Study Institutes Series, pages 185-213. Springer US, 1981.

[6] H. L. Cycon, R. G. Froese, W. Kirsch, and B. Simon. Schrödinger operators with application to quantum mechanics and global geometry. Texts and Monographs in Physics. Springer-Verlag, Berlin, study edition, 1987.

[7] M. Dauge and N. Raymond. Plane waveguides with corners in the small angle limit. Journal of Mathematical Physics, 53(12):123529, 2012.

[8] M. Dimassi and J. Sjöstrand. Spectral asymptotics in the semi-classical limit, volume 268 of London Mathematical Society Lecture Note Series. Cambridge University Press, Cambridge, 1999.

[9] S. Fournais and B. Helffer. Spectral methods in surface superconductivity. Progress in Nonlinear Differential Equations and their Applications, 77. Birkhäuser Boston Inc., Boston, MA, 2010.

[10] P. Freitas. Precise bounds and asymptotics for the first Dirichlet eigenvalue of triangles and rhombi. J. Funct. Anal., 251(1):376-398, 2007.

[11] L. Friedlander and M. Solomyak. On the spectrum of the Dirichlet Laplacian in a narrow strip. Israel J. Math., 170:337-354, 2009.

[12] B. Helffer. Semi-classical analysis for the Schrödinger operator and applications, volume 1336 of Lecture Notes in Mathematics. Springer-Verlag, Berlin, 1988.

[13] B. Helffer and J. Sjöstrand. Multiple wells in the semiclassical limit. I. Comm. Partial Differential Equations, 9(4):337-408, 1984.

[14] L. Hillairet and C. Judge. Spectral simplicity and asymptotic separation of variables. Comm. Math. Phys., 302(2):291-344, 2011.

[15] T. Kato. Perturbation theory for linear operators. Die Grundlehren der mathematischen Wissenschaften, Band 132. Springer-Verlag New York, Inc., New York, 1966.

[16] M. Klein, A. Martinez, R. Seiler, and X. P. Wang. On the Born-Oppenheimer expansion for polyatomic molecules. Comm. Math. Phys., 143(3):607-639, 1992.

[17] V. A. Kondrat'ev. Boundary value problems for elliptic equations in domains with conical or angular points. Trudy Moskov. Mat. Obšč., 16:209-292, 1967.

[18] Y. Lafranche and D. Martin. MÉLINA++, bibliothèque de calculs éléments finis. http://anum-maths.univ-rennes1.fr/melina/, 2012.

[19] Z. Lu and J. Rowlett. The fundamental gap of simplices. Comm. Math. Phys., 319(1):111-145, 2013.

[20] A. Martinez. Développements asymptotiques et effet tunnel dans l'approximation de BornOppenheimer. Ann. Inst. H. Poincaré Phys. Théor., 50(3):239-257, 1989. 
[21] B. J. McCartin. Eigenstructure of the equilateral triangle. I. The Dirichlet problem. SIAM Rev., 45(2):267-287 (electronic), 2003.

[22] T. Ourmières-Bonafos. Dirichlet eigenvalues of cones in the small aperture limit. Journal of Spectral Theory, To appear.

[23] M. Reed and B. Simon. Methods of modern mathematical physics. IV. Analysis of operators. Academic Press [Harcourt Brace Jovanovich Publishers], New York, 1978.

[24] B. Simon. Semiclassical analysis of low lying eigenvalues. I. Nondegenerate minima: asymptotic expansions. Ann. Inst. H. Poincaré Sect. A (N.S.), 38(3):295-308, 1983. 